SLAC-PUB-13225

April 2008

\title{
Lattice and Collective Effects for PEP-X *
}

Karl Bane, Yunhai Cai, Alex Chao, Robert Hettel, Zhirong Huang, Yuri Nosochkov, Gennady Stupakov, Lanfa Wang, and Min-Huey Wang

Stanford Linear Accelerator Center

Stanford University, Stanford, CA 94309

\begin{abstract}
This is a more comprehensive report of the accelerator physics in the white paper "PEP-X Light Source at SLAC". A new light source called "PEP-X" would reside in the 2.2-km PEP-II tunnel. It has a hybrid lattice where two of its six arcs contain DBA cells that provide a total of 30 straight sections for insertion device beam lines and the remaining arcs contain TME cells for an extremely low emittance. Using 90 meter damping wigglers the horizontal emittance at $4.5 \mathrm{GeV}$ is further reduced to $0.1 \mathrm{~nm}$-rad. Many collective effects including intra-beam scattering, Touschek lifetime, and fast ion instability are studied. We expect that PEP-X will produce photon beams having brightnesses near $10^{22}\left(\mathrm{ph} / \mathrm{s} / \mathrm{mm}^{2} / \mathrm{mrad}^{2} / 0.1 \% \mathrm{BW}\right)$ at 10 $\mathrm{keV}$ and $10^{21}$ at $35 \mathrm{keV}$.
\end{abstract}

* Work supported by the Department of Energy under Contract No. DE-AC02-76SF00515. 


\section{INTRODUCTION}

Our primary design goals are:

- Achieving a very low emittance beam of about $0.1 \mathrm{~nm}$-rad at an energy of 4.5 $\mathrm{GeV}$ (not including the effect of intra-beam scattering)

- Providing adequate dynamic aperture to accept the electron beam from the existing PEP-II[1] injector

- Storing high beam current up to 1.5 A stably and with adequate lifetime

- Providing at least 24 short-straight and dispersion-free regions in which to place the undulator insertion devices (ID) and maintaining flexibility to change its nearby optics

- Fitting the ring into the existing PEP-II tunnel and using its injector, and RF system

To achieve these challenging goals, we have introduced the following features into our design:

- Theoretical minimum emittance[2] (TME) cells to achieve the very low emittance

- Double bend achromat[3] (DBA) cells to provide spaces for IDs and to retain emittance

- 90-meter damping wigglers to further reduce the emittance and damping time 
- A powerful and low emittance injector to allow us to continuously inject electrons into the ring and to tolerate somewhat smaller acceptance of the ring.

- Double the number of bunches to $n_{b}=3400$ to mitigate the effects of intrabeam scattering, Touschek lifetime, and other single-bunch instabilities

- A large number of RF buckets to enable us to have flexible bunch patterns to mitigate the effects due to fast ion instability (FII)

In this paper, we will give a general overview of the design and discuss some critical issues of accelerator physics associated with the design. First, we will have a comprehensive description of the layout and optics of the lattice. Then, we will present a study of a dynamic aperture and injection scheme. In order to have a realistic beam emittance and lifetime, we will follow up with calculations of emittance growth due to the intra-beam scattering and an estimation of the Touschek lifetime. Finally, we will provide a preliminary estimate of thresholds and growth rates of collective instabilities including the fast ion instability.

\section{LATTICE DESIGN}

The PEP-X layout is shown in Fig. 1. For a proper fit in the existing tunnel, the design adopts the same ring geometry as in PEP-II with the six arcs and six long straight sections of the same length and the same circumference of 2199.32 m. Geometric positions of the straight sections are identical to those in PEP-II, but there are small variations of the radial positions inside the arcs due to the new locations and strengths of the PEP-X bending magnets.

The two PEP-II rings, HER and LER, have the same circumference and similar 


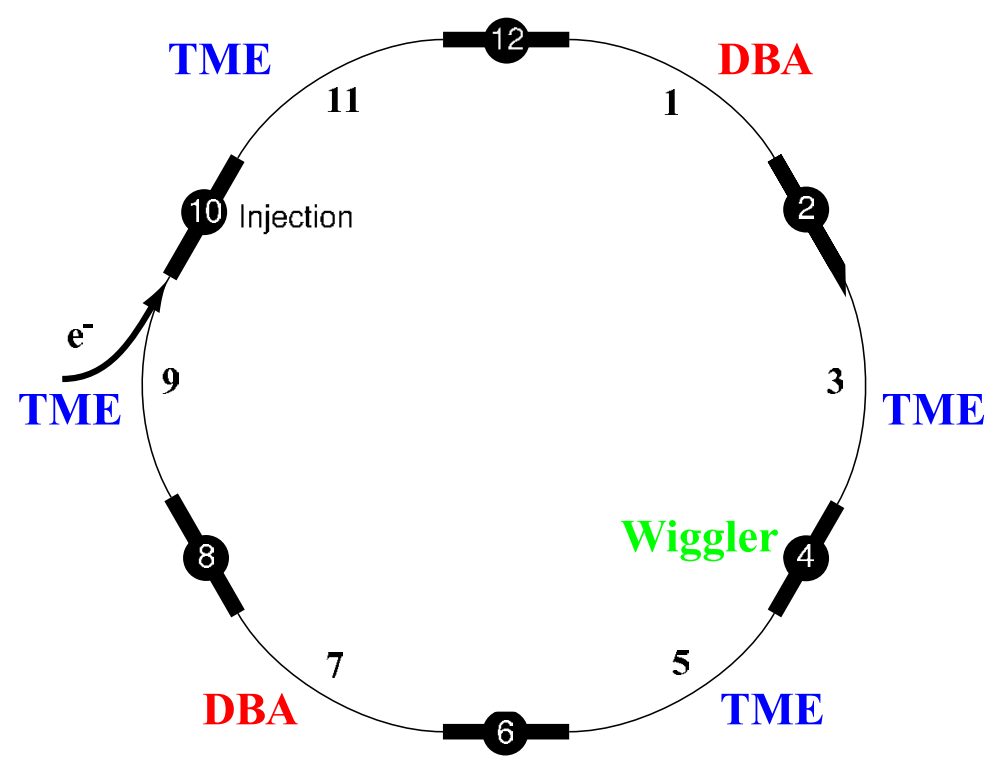

FIG. 1: PEP-X layout with 6 straight sections, 2 DBA arcs and 4 TME arcs.

optics. However, the HER can provide a much lower emittance since it uses 12 times longer bending magnets in the FODO arcs. This is an advantage if the upgrade to PEP-X is done in several stages, where some of the existing arcs are used in the intermediate stages. For this reason, the HER is selected for conversion to PEP-X.

The FODO arcs in the present HER are not suitable for PEP-X since they do not provide space for IDs, and their lowest possible emittance is two orders of magnitude higher than the PEP-X goal. To accommodate a large number of insertion devices, the FODO cells in two arcs will be replaced with the DBA cells. The other four arcs will be converted to the TME lattice and $\sim 90 \mathrm{~m}$ damping wiggler will be added to a long straight section in order to achieve the desired low emittance.

Since the length of PEP-X arc is fixed at $243.2 \mathrm{~m}$, the choice of the number of DBA cells per arc is a compromise between the cell length and its optical and 
physical properties. The main advantage of a short cell is that the arc can accommodate more insertion devices. The shorter cell also uses a smaller bending angle $\theta$ in dipoles and therefore can achieve a lower emittance since it scales as $\epsilon_{x} \propto \theta^{3}$. The disadvantage is that the space for ID and the magnets is reduced which may limit the optics flexibility. The other factors which determine the DBA properties are the requirement for dispersion cancellation within each cell and the choice of a phase advance. Two options, with 16 and 12 DBA cells per arc, have been compared. Here, the number of cells is chosen to be a multiple of 4 which in combination with the cell phase advance of $\pi / 2+n \pi$ provides conditions for cancellation of chromatic and sextupole aberrations locally in every 4 cells. It was found that the only advantage of the 12 cell option is the longer $6 \mathrm{~m}$ ID straights as compared to $4.26 \mathrm{~m}$ straights in the 16 DBA option. The beam optical acceptance and the predicted photon brightness in these options are rather similar. Since the higher number of IDs per arc leads to a more cost efficient design, the 16 DBA option has been adopted.

The layout and optics functions for one $15.21 \mathrm{~m}$ DBA cell are shown in Fig. 2. The cell has a symmetric optics with 6 quadrupoles, 3 two family sextupoles and two $1.0 \mathrm{~m}$ combined function dipoles. The latter improve optics flexibility due to the extra vertical focusing. The dispersion free space in the beginning and end of the cell, where $\beta_{x}=9.1 \mathrm{~m}, \beta_{y}=8.1 \mathrm{~m}$, is reserved for the insertion devices. The optics is optimized for a low emittance value, the lowest ID $\beta$ functions and the lowest strengths of sextupoles. The cell phase advance is slightly detuned to $\mu_{x} / 2 \pi=0.7366$ and $\mu_{y} / 2 \pi=0.2376$ to improve the ring chromatic compensation properties and dynamic aperture. In total, the two DBA arcs will contain thirty $4.26 \mathrm{~m}$ straights for $3 \mathrm{~m}$ IDs and four $1.90 \mathrm{~m}$ straights at the arc ends. The first and the last cells in the DBA arc are shortened by $8 \mathrm{~cm}$ for a proper fit to the existing 

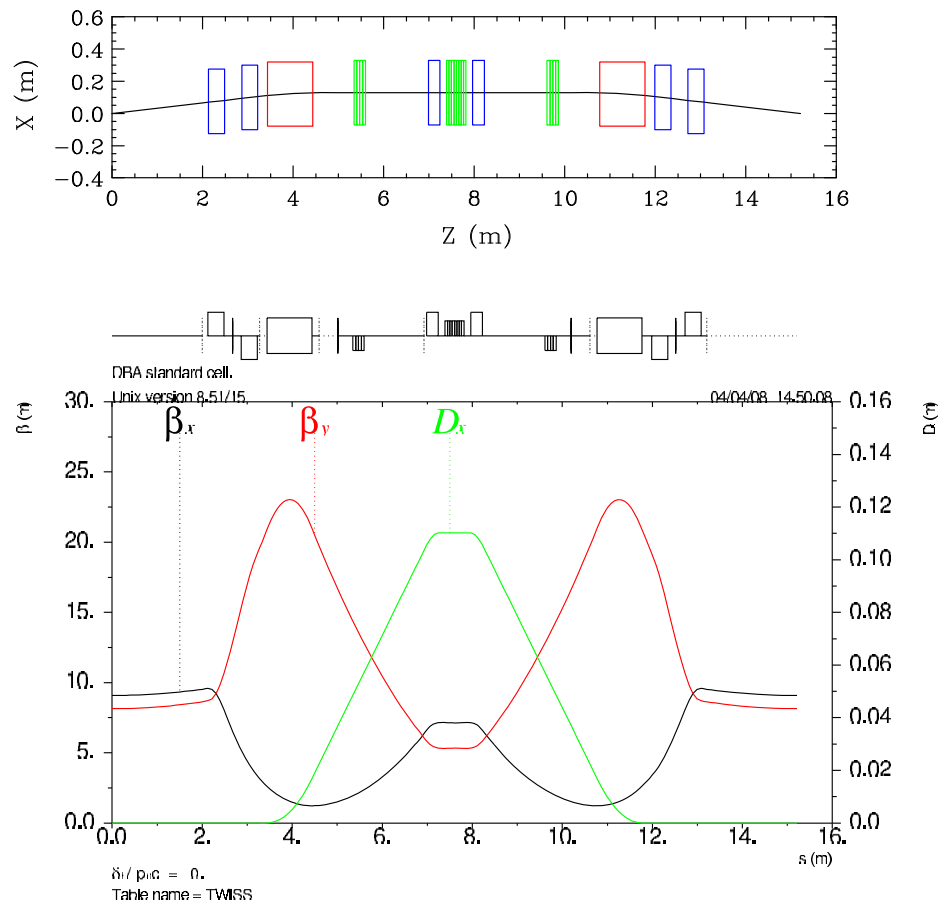

FIG. 2: Layout and optics functions in one DBA cell.

HER geometry, and the two quads at each arc end are used for $\beta$ matching to the long straight sections.

The other four arcs will contain the TME optics which is the superior lattice for reaching the lowest emittance. Each TME arc will contain 32 regular and 2 matching cells. Due to the non-zero dispersion and limited free space, the TME cell is not suitable for insertion devices. The layout and optics functions for one $7.297 \mathrm{~m}$ regular cell are shown in Fig. 3. The cell symmetric optics uses 4 two family quadrupoles, 3 two family sextupoles and one $2.7 \mathrm{~m}$ dipole. The TME low emittance property is due to the optimal dipole location at the waist of the horizontal $\beta$ function and dispersion at center of the cell. The chosen high number of short cells decreases 
the dipole bending angle and therefore reduces the emittance $\epsilon_{x} \propto \theta^{3}$. Positions of the two family sextupoles are optimized for the most orthogonal correction of $x$ and $y$ chromaticity leading to the lowest possible sextupole strengths. The cell phase advance is $\mu_{x} / 2 \pi=0.375$ and $\mu_{y} / 2 \pi=0.125$ which provides conditions for local cancellation of chromatic and sextupole aberrations in every 8 cells.
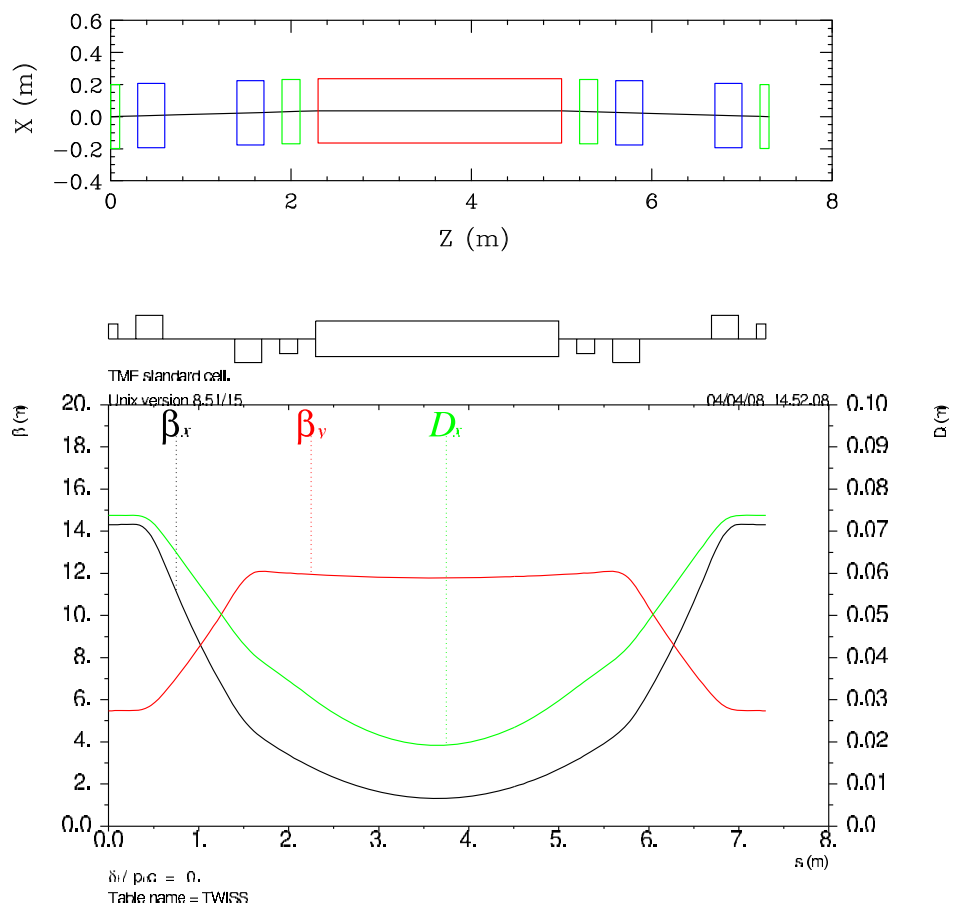

FIG. 3: Layout and optics functions in one regular TME cell.

The TME arc has a special matching cell at each arc end which is designed to cancel the dispersion, help with a $\beta$ match and fit the arc geometry to the existing HER ring. Fig. 4 shows the layout and optics functions in the matching cell and the next two regular cells in the beginning of the TME arc. The optimized matching cell is $4.846 \mathrm{~m}$ long and it uses 2 quadrupoles and a $35 \%$ shorter dipole than in a 
regular cell.
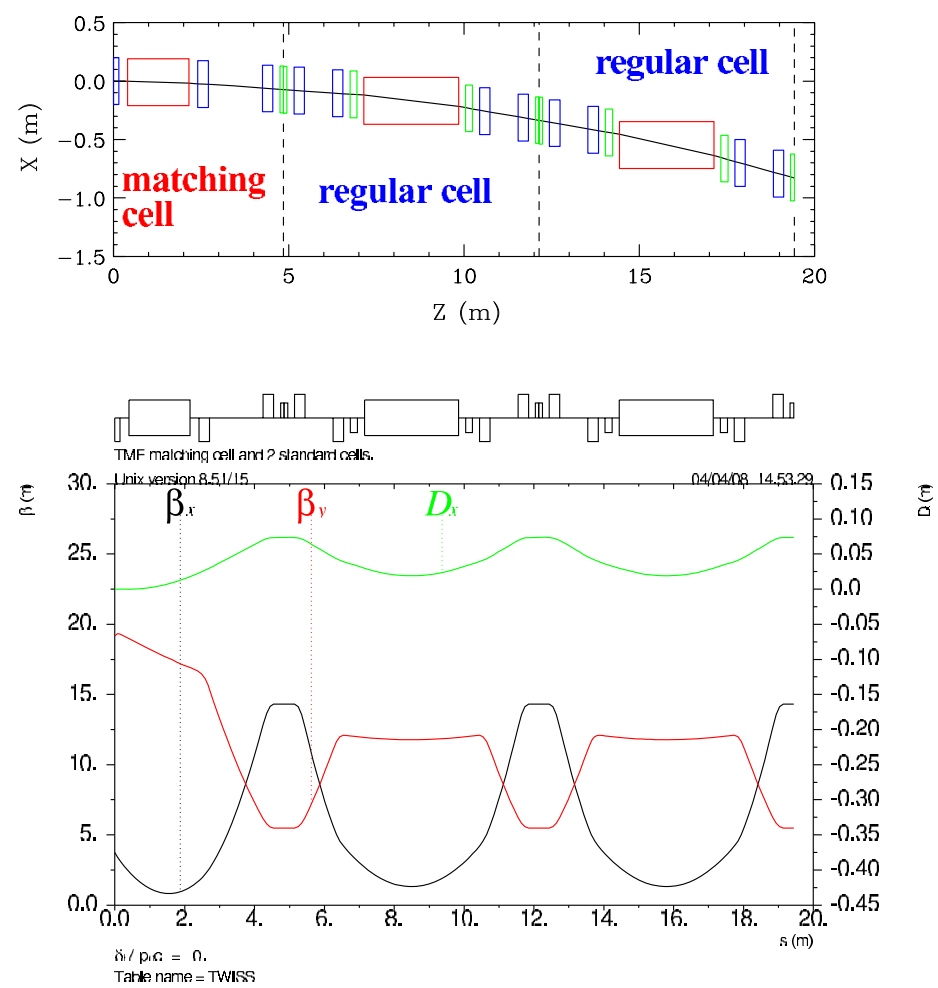

FIG. 4: Layout and optics functions in the matching and two regular TME cells.

The PEP-X straight sections will maintain the same position and length (123.353 m) as in the PEP-II HER. They will contain the injection system, the RF accelerating cavities, the damping wiggler and will be used for betatron tune adjustment. In the PEP-X design, 5 straight sections have an identical FODO lattice with 21 quadrupoles as shown in Fig. 5. The quadrupole strengths in each straight depend on the type of adjacent arcs (DBA or TME) and the presence of the damping wiggler. The 4 cells in the middle of the straight use two family quadrupoles and have exactly periodic optics functions. Together with the six family quadrupoles at either 
end of the straight they provide optical match to the DBA and TME arcs as well as adjustment of betatron tune. The first and the last focusing quadrupoles are moved closer to the beginning and end of the straight in order to minimize the matched $\beta$ functions.

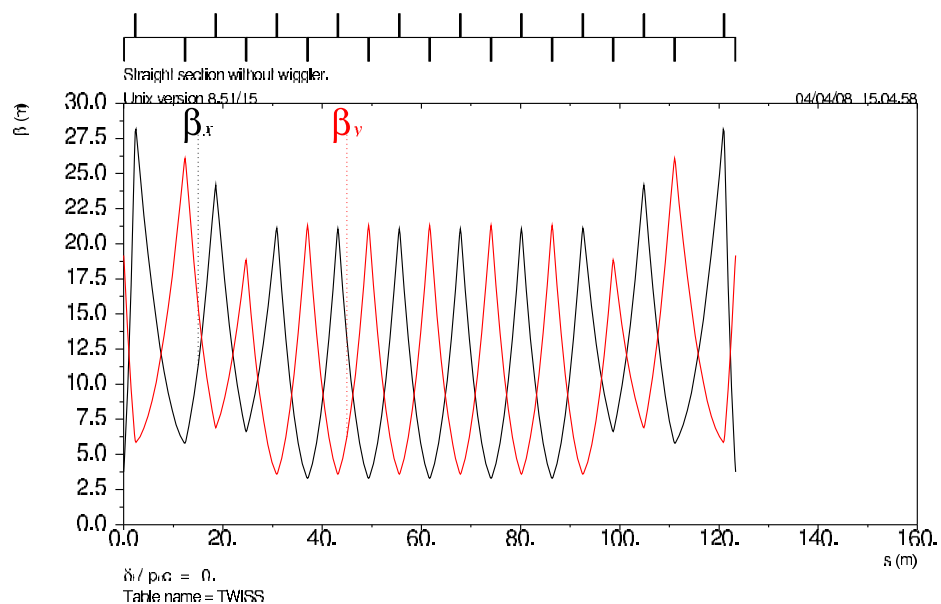

FIG. 5: Optics functions in the FODO straight section without wiggler.

The PEP-X DBA and TME optics attains horizontal emittance of $0.37 \mathrm{~nm}$ at 4.5 $\mathrm{GeV}$. Further reduction to the level of $0.1 \mathrm{~nm}$ requires a strong damping wiggler. In this design, the wiggler is inserted in the straight section 4 as shown in Fig. 1. However this straight may also be appropriate for placing the RF cavities. In this case, the wiggler could be accommodated in straights 2 and 6 without a significant change to the beam parameters. Layout of the FODO straight section with the damping wiggler is shown in Fig. 6. The total wiggler length is $89.325 \mathrm{~m}$, which is split into 18 identical sections, each $4.9625 \mathrm{~m}$ long, inserted between the quadrupoles. For a strong damping effect, the wiggler has a short $10 \mathrm{~cm}$ period and optimal 1.5 $\mathrm{T}$ vertical field. Fig. 7 shows one $4.9625 \mathrm{~m}$ wiggler section containing 50 periods and the corresponding horizontal dispersion which is canceled outside of the wiggler. 
The wiggler also creates a periodic horizontal orbit which has the same form and amplitude as the dispersion.

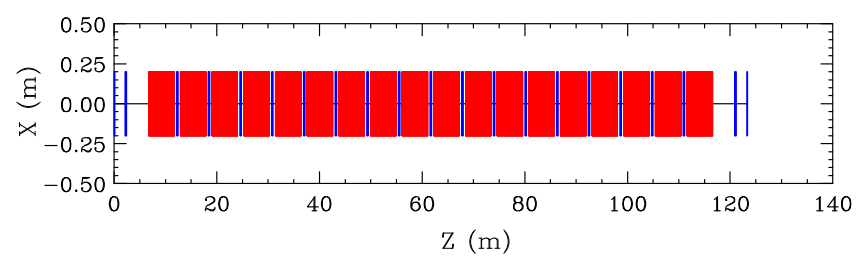

FIG. 6: $89.325 \mathrm{~m}$ wiggler (red) in the FODO straight section.

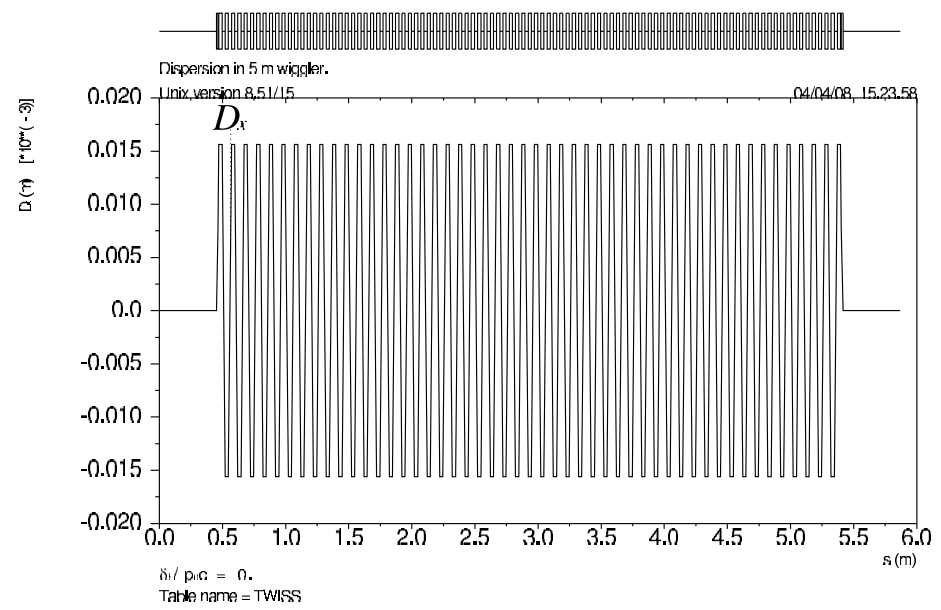

FIG. 7: Horizontal dispersion in the $4.9625 \mathrm{~m}$ wiggler section.

The present design uses a vertical injection into PEP-X because of the larger beam vertical acceptance. The injection section adopts the existing HER magnet and kicker configuration, but adds 4 additional quadrupoles at the straight ends for improved $\beta$ matching to the TME arcs. The magnet layout and optics functions in the injection section are shown in Fig. 8. The injection point is at center of the straight where a high vertical beta function $\beta_{y}=200 \mathrm{~m}$ is used. The latter increases 
the vertical size of the stable beam area and therefore provides more room for the injected beam. Further enlargement of the injection acceptance is achieved by moving the stored beam close to the injection septum using the DC bump magnets and fast kickers shown in Fig. 8. The four DC magnets can control both the amplitude and angle of the stored beam closed bump, and the two identical kickers, separated by $180^{\circ}$ vertical phase advance, provide an additional symmetric bump.
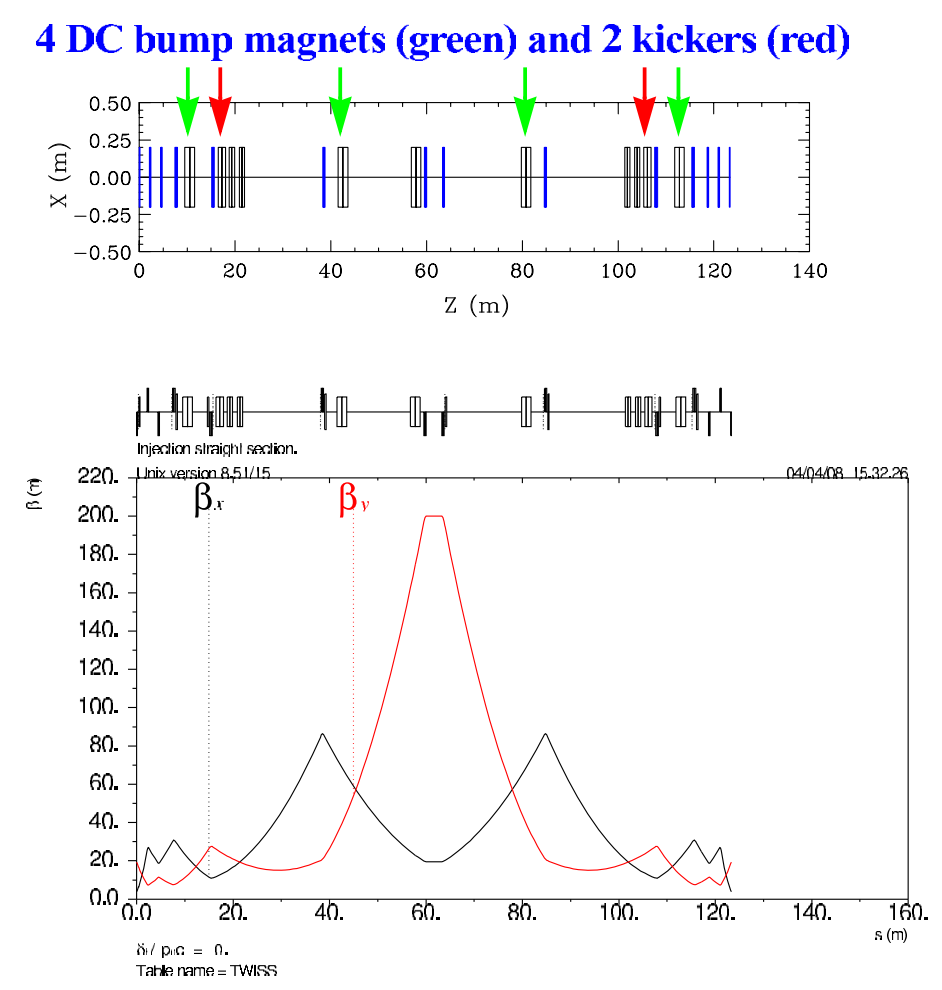

FIG. 8: Layout and optics functions in the injection section.

Optics functions in the complete PEP-X ring are shown in Fig. 9. The order of the DBA and TME arcs in the ring is chosen to be symmetric, but depending on practical considerations, it can be changed without affecting the main beam parameters. The 
PEP-X betatron tune dependence on relative momentum error $\Delta p / p$ is shown in Fig. 10. It shows that compensation of chromatic tune variation is very good for a range of $\Delta p / p= \pm 2 \%$. This helps to maximize the optical momentum acceptance by keeping the off-momentum particles well within the design tune area and away from strong resonances.

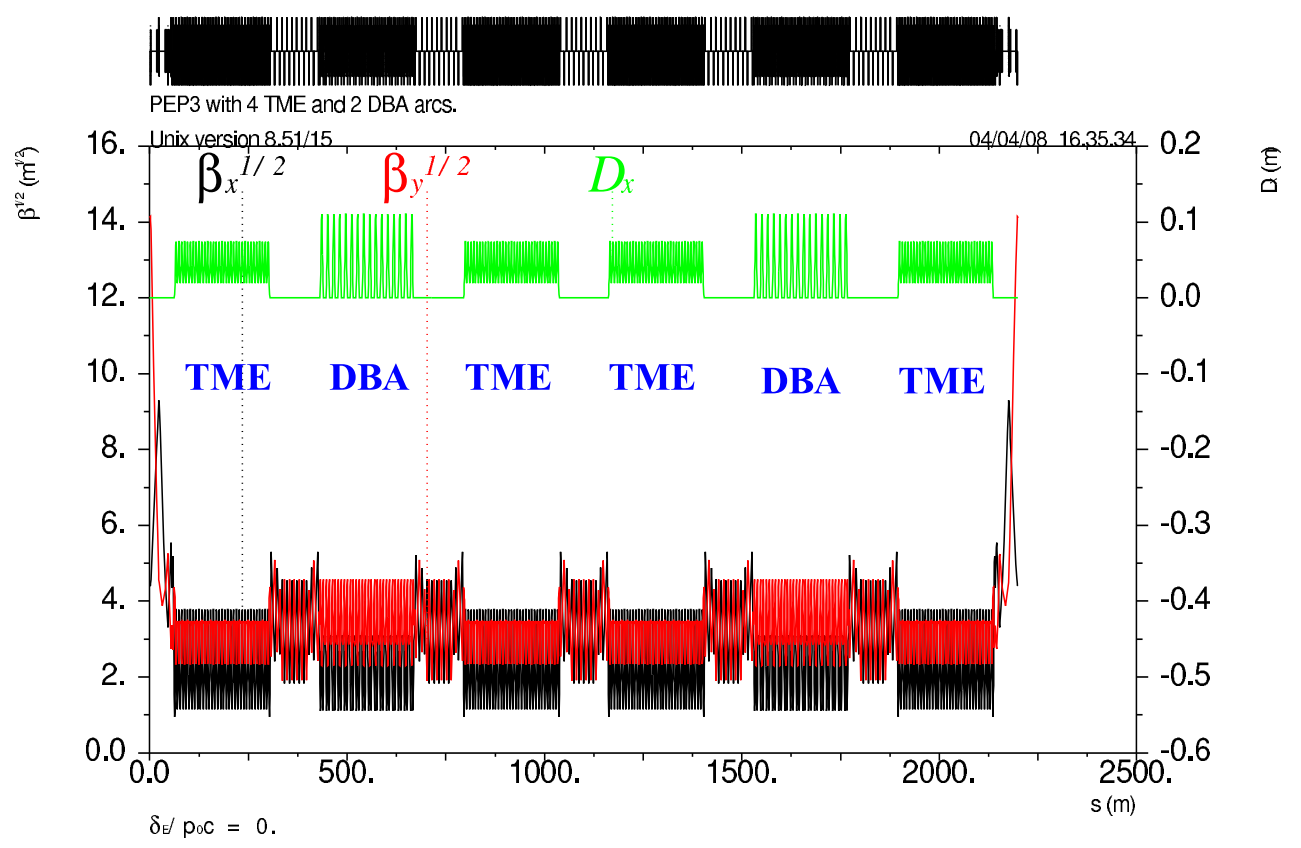

FIG. 9: Optics functions in the complete PEP-X ring.

The PEP-X lattice parameters are listed in Table I. The $0.094 \mathrm{~nm}$ emittance value listed does not include the emittance growth due to intra-beam scattering (IBS) which will be discussed in a separate section.

The number of PEP-X dipole, quadrupole and sextupole magnets is shown in Table II. The highly packed TME arcs contain most of the new magnets. At the present stage, the design does not include a detailed configuration of magnet 


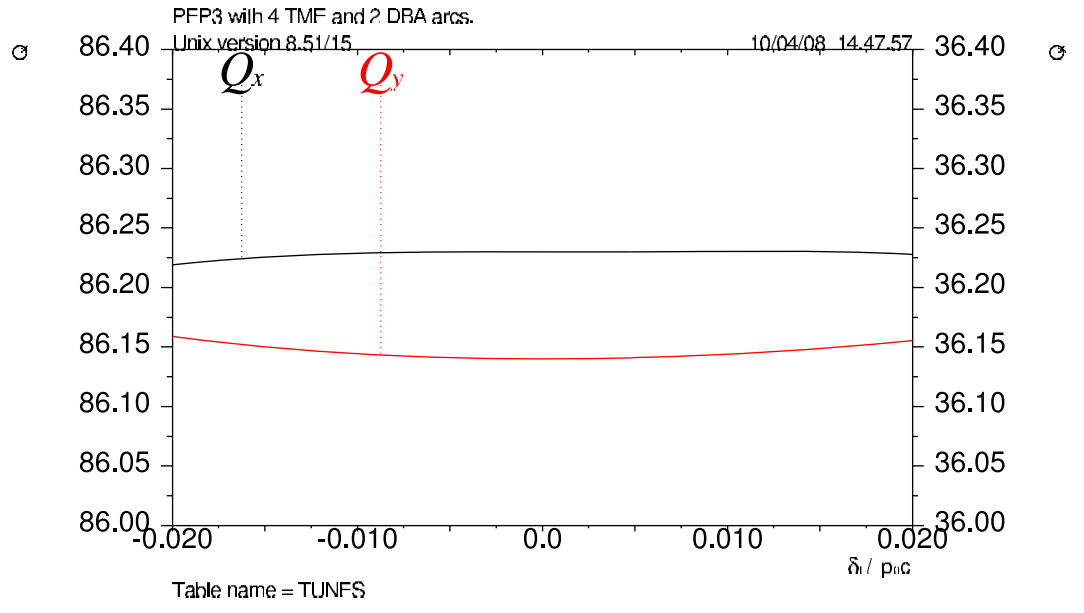

FIG. 10: PEP-X betatron tune as a function of relative momentum error $\Delta p / p$.

correctors and beam position monitors (BPM). However, there will be approximately one corrector and one BPM per quadrupole. The estimated number of independent power supplies is 2 for DBA and TME dipoles, 164 for quadrupoles and 4 for DBA and TME two family sextupoles. The large number of quadrupole supplies is due to the assumption that all insertion devices in the DBA arcs will be different and therefore require a different optical match. The current design does not use the existing PEP-II magnets, except in the injection section. However, in the future, the lattice can be optimized for utilizing some of the existing magnets. 
TABLE I: PEP-X lattice parameters.

\begin{tabular}{|l|c|}
\hline Energy, GeV & 4.5 \\
\hline Circumference $[\mathrm{m}]$ & 2199.32 \\
\hline Betatron tune, $x / y$ & $86.23 / 36.14$ \\
\hline Synchrotron tune & 0.00742 \\
\hline Momentum compaction & $4.72 \cdot 10^{-5}$ \\
\hline Emittance without IBS $[\mathrm{nm}]$ & 0.094 \\
\hline RMS bunch length $[\mathrm{mm}]$ & 2.50 \\
\hline RMS momentum spread & $1.12 \cdot 10^{-3}$ \\
\hline Damping time, $x / y / s[\mathrm{~ms}]$ & $19.7 / 20.2 / 10.2$ \\
\hline Natural chromaticity, $x / y$ & $-132.7 /-72.8$ \\
\hline Energy loss $[$ MeV $/$ turn] & 3.27 \\
\hline RF voltage $[\mathrm{MV}]$ & 10 \\
\hline Total wiggler length $[\mathrm{m}]$ & 89.325 \\
\hline Wiggler period $[\mathrm{m}]$ & 0.1 \\
\hline Wiggler field $[\mathrm{T}]$ & 1.5 \\
\hline Regular ID straight length $[\mathrm{m}]$ & 4.26 \\
\hline Number of regular ID straights & 30 \\
\hline$\beta_{x} / \beta_{y}$ at ID center $[\mathrm{m}]$ & $9.09 / 8.14$ \\
\hline
\end{tabular}


TABLE II: PEP-X magnet quantity.

\begin{tabular}{|l|c|}
\hline $1.0 \mathrm{~m}$ DBA combined function bends & 64 \\
\hline $2.7 \mathrm{~m}$ TME regular bends & 128 \\
\hline 1.755 m TME matching bends & 8 \\
\hline Total bending magnets & $\mathbf{2 0 0}$ \\
\hline \hline DBA cell quadrupoles & 192 \\
\hline TME regular cell quadrupoles & 512 \\
\hline TME matching cell quadrupoles & 16 \\
\hline FODO straight quadrupoles & 105 \\
\hline Injection straight quadrupoles & 14 \\
\hline Total quadrupoles & $\mathbf{8 3 9}$ \\
\hline \hline DBA cell sextupoles & 96 \\
\hline TME cell sextupoles & 384 \\
\hline Total sextupoles & $\mathbf{4 8 0}$ \\
\hline
\end{tabular}




\section{DAMPING WIGGLERS}

For a modern light source design the damping wigglers play an important part in obtaining higher brightness. Compared to the other method to reduce the emittance using damping wigglers is an efficient way. With optimal choice of the parameters of the damping wiggler, the emittance can be brought to a very small value. The horizontal emittance variation due to the damping wigglers in the dispersion free straight is given as[4]:

$$
\frac{\epsilon_{w}}{\epsilon_{0}}=\frac{1+\frac{4 C_{q}}{15 \pi J_{x}} N_{p} \frac{\leq \beta_{x}>}{\epsilon_{0} \rho_{w}} \gamma^{2} \frac{\rho_{0}}{\rho_{w}} \theta_{w}^{3}}{1+\frac{1}{2} N_{p} \frac{\rho_{0}}{\rho_{w}} \theta_{w}},
$$

where $C_{q}=3.81 \times 10^{-13} \mathrm{~m},<\beta_{x}>$ is the average horizontal beta function in the wiggler, $N_{p}$ is the total number of wiggler periods, $\rho_{w}$ is the wiggler bending radius at the peak field, $\theta_{w}=\lambda / 2 \pi \rho_{w}$ is the peak trajectory angle in the wiggler, $\lambda_{w}$ is the wiggler period length, $\rho_{0}$ is the bending radius of the ring dipole, and $\epsilon_{0}$ is the emittance without the damping wiggler. The wigglers also cause the energy spread to grow:

$$
\frac{\sigma_{E}}{E} \approx\left(\frac{\sigma_{E}}{E}\right)_{0} \sqrt{\frac{1+\frac{2 L_{w} \rho_{0}^{2}}{3 \pi^{2} \rho^{3}}}{1+\frac{L_{w} \rho_{0}}{4 \pi \rho_{w}^{2}}}},
$$

where $L_{w}=N_{p} \lambda_{w}$ is the total length of the damping wiggler.

The ability of the damping wiggler to reduce the emittance is determined by the period length of the wiggler, the strength of peak wiggler field, and the total wiggler length. The emittance variations of the damping wiggler as a function of wiggler field strength with different wiggler period lengths based on the PEP-X lattice are shown in Fig. 11. The effects of emittance reduction versus total wiggler length of different wiggler periods are shown in Fig. 12. The beam emittance without the 
TABLE III: Parameters of damping wigglers for PEP-X, $<\beta_{x}>=10.34 \mathrm{~m}, J_{x}=1, \epsilon_{0}=0.37$ nm-rad, and $L_{w}=89.3 \mathrm{~m}$.

\begin{tabular}{|c|c|c|c|c|c|}
\hline$\lambda_{w}(\mathrm{~cm})$ & $B_{w}(\mathrm{~T})$ & $\epsilon_{w} / \epsilon_{0}$ & Full gap $(\mathrm{mm})$ & $\mathrm{K}$ & $\lambda_{r}(\AA)$ \\
\hline 10 & 1.5 & 0.32 & 15.4 & 14.1 & 647 \\
\hline \multicolumn{7}{|c|}{ Other options to accomodate a soft x-ray FEL undulator } \\
\hline 5 & 1.27 & 0.36 & 9.39 & 5.93 & 60.0 \\
\hline 5 & 0.5 & 0.78 & 20.0 & 2.33 & 12.0 \\
\hline \multicolumn{7}{|c|}{ Superconducting magnet } \\
\hline 1.4 & 1.5 & 0.30 & 5.0 & 1.96 & 2.64 \\
\hline
\end{tabular}

damping wiggler is $0.37 \mathrm{~nm}$-rad in the PEP-X storage ring. The average horizontal beta function is $10.34 \mathrm{~m}$ and the horizontal damping partition number is 1 . Due to the small initial emittance, the parameters of the damping wiggler incline to be a short wiggler period and a high wiggler field as shown in Fig. 11. The installation of the damping wiggler is to reduce the beam emittance from $0.37 \mathrm{~nm}$-rad to 0.1 nm-rad. The wiggler period of $10 \mathrm{~cm}$, maximum field of 1.5 Tesla and total length of $89.3 \mathrm{~m}$ are chosen as the parameters of the damping wiggler of PEP-X. The emittance is reduced to $0.094 \mathrm{~nm}-\mathrm{rad}$ as calculated by MAD8[5]. The parameters of damping wigglers are shown in Table III.

A discussion to combine the damping wigglers with a soft x-ray FEL undulator is also shown in the middle part of the table. Preliminary studies[6] show that for an ultra-low emittance, high peak current PEP ring, FEL exponential gain (without saturation) at soft x-ray wavelengths can occur on a turn-by-turn basis, with only a very modest degradation in beam energy spread. In the last line of the table, 
using super conduction undulators is also considered to take advantage of future accelerator technology.

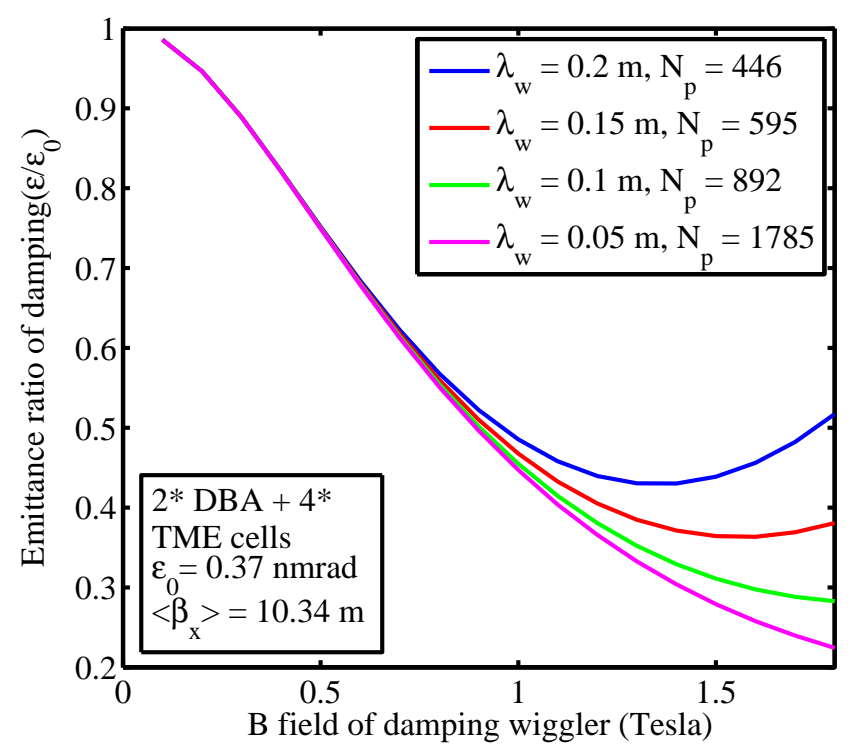

FIG. 11: Emittance reduction of the damping wiggler as a function of wiggler field strength with different wiggler period length. The total wiggler length is $89.3 \mathrm{~m}$. The beam emittance without the damping wiggler is $0.37 \mathrm{~nm}$-rad. The average horizontal beta function is 10.34 $\mathrm{m}$ and the horizontal damping partition number is 1 .

The installation of the damping wiggler can cause other effects. A strong wiggler field and short period length imply a small wiggler gap as shown in Fig. 13 for normal magnet design. This will put a constraint on the vertical aperture in the long straight and usually reduces the beam life time. The energy spread will increase from $6.1 \times 10^{-4}$ to $1.12 \times 10^{-3}$. The radiation energy $U_{0}$ per electron per revolution is increased from $0.68 \mathrm{MeV}$ to $3.273 \mathrm{MeV}$. For a total beam current of $3 \mathrm{~A}$, the radiation 
power of the damping wigglers is 7.78 MW. This will require extra RF power and special care to deal with the heat of the radiated power. A simple estimate of power handling indicates that we may need to break up the 90-meter wigglers into two separated sections. The effects of the nonlinear field of the damping wigglers on beam dynamics will need to be evaluated, which is not included in this report.

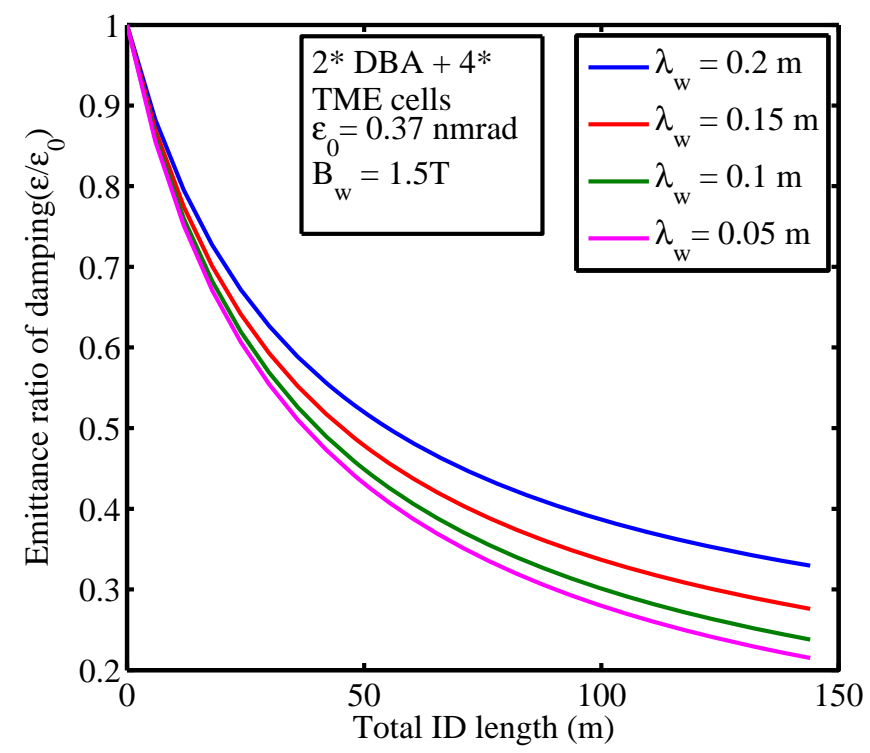

FIG. 12: Effects of emittance deduction versus total wiggler length of different wiggler periods.

\section{DYNAMIC APERTURE}

For a small emittance light source design strong focusing is inevitable. Therefore strong sextupoles to correct the natural chromaticity to zero or slightly positive to 


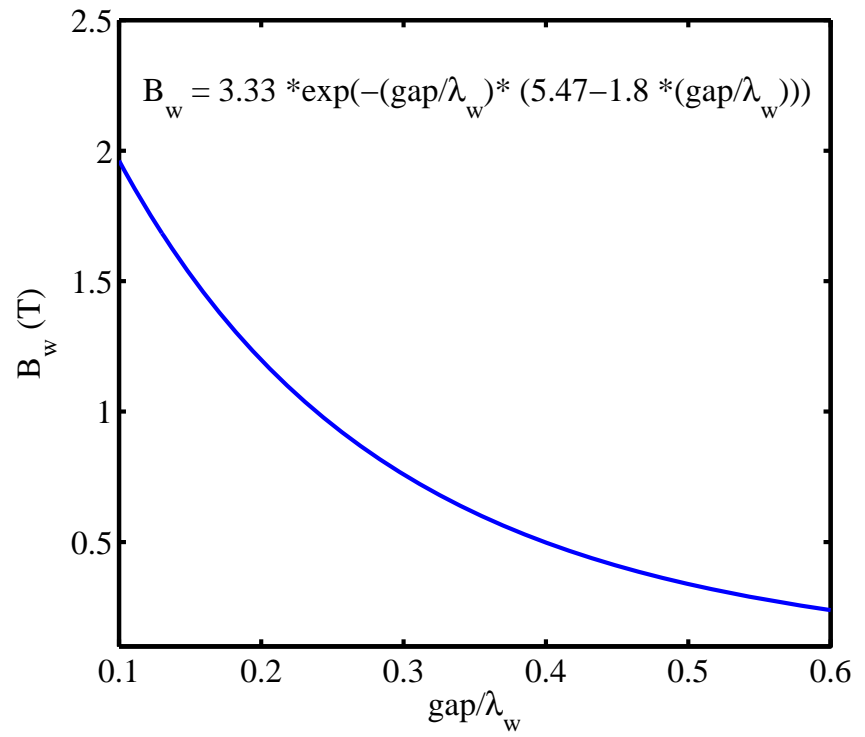

FIG. 13: Wiggler field strength for hybrid magnet design for small gap.

overcome the transverse head tail instability is needed. The nonlinear effects driven by such strong chromatic sextupoles can result in a severely decreased dynamic aperture. Achieving a large dynamic aperture is basically the process of canceling out the effects of these nonlinear kicks. The arrangement of the sextupoles is in essence to minimize the strength of the sextupole by putting sextupoles at the locations where the dispersion is large and the beta functions are well separated. The phase advance of unit cell is also chosen to cancel out the first order terms. The phase advances per TME cell are $\mu_{x} / 2 \pi=0.1875$ and $\mu_{y} / 2 \pi=0.0625$ for horizontal and vertical respectively. The phase advances per DBA cell are $\mu_{x} / 2 \pi=0.7366$ and $\mu_{y} / 2 \pi=0.2376$ respectively. A dynamic aperture search of scans of global tunes by adjusting the quadrupoles strength of FODO in the long straight section are shown 
in Fig. 14. The working tunes of 86.23 and 36.14 are chosen. There are four families of sextupoles. One pair named SD, SF is in the TME cell and the other pair SD1, SF1 is in the DBA cell. The linear chromaticity is corrected to zero. The higher order chromatic tune is shown in Fig 10. The dynamic aperture tracking of the bare lattice is shown in Fig. 15. The tracking point is set at the injection point. A 3 $\sigma$ injected beam with injected beam emittance and $\beta$ of storage ring is shown in Fig. 15. The vertical dynamic aperture is sufficient for the vertical injection. The dynamic aperture tracking result with systematic and random multipole errors is also shown in Fig. 16.
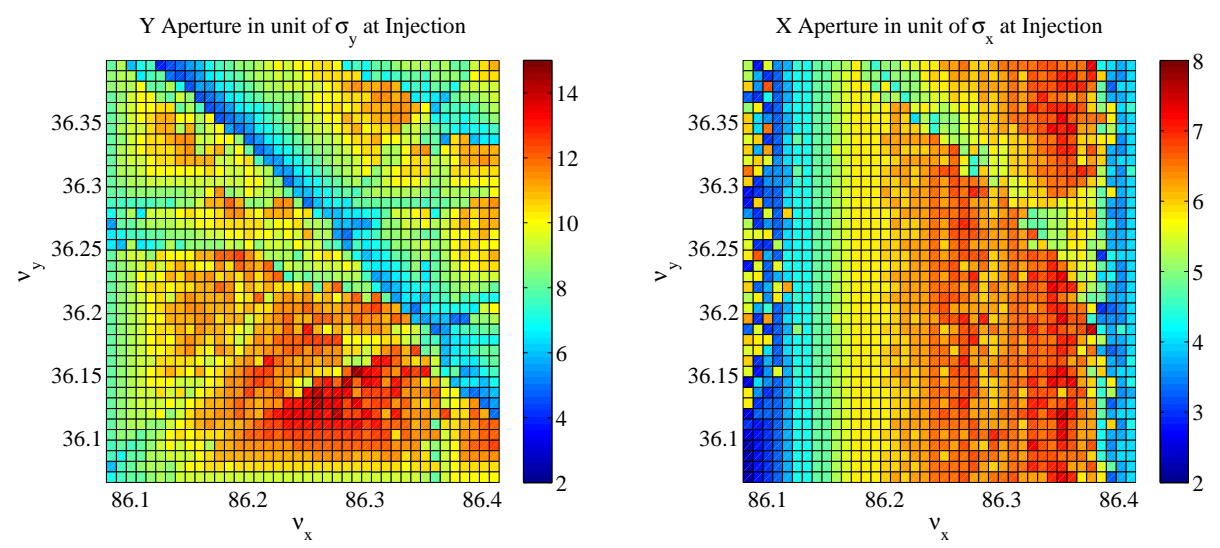

FIG. 14: Dynamics aperture scan of different working tunes.

\section{INJECTION}

The injection scheme of PEP-X will adapt the design of PEP-II at the present i.e. injection from the vertical plane. The stored beam will be bumped by four DC bump magnets and then kicked by two identical pulse kickers, with 180 degree 


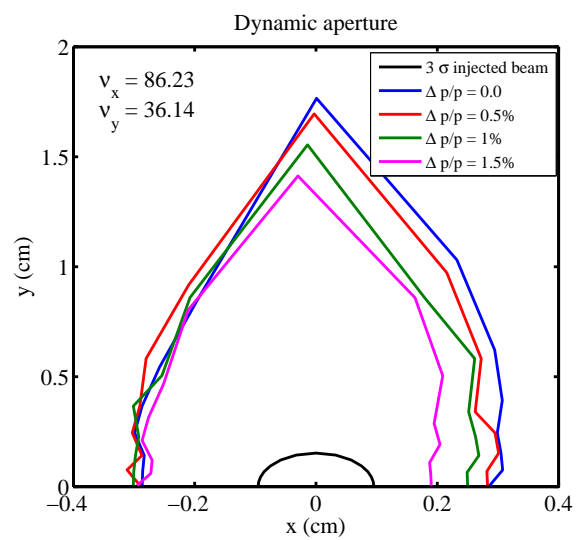

FIG. 15: Dynamic aperture tracking of the bare lattice.

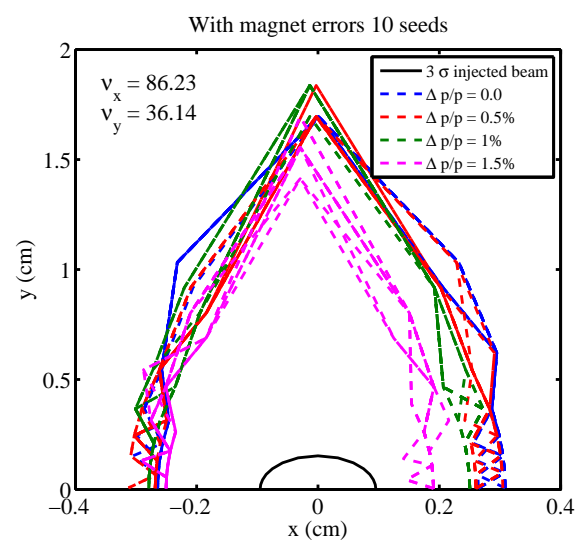

FIG. 16: Dynamic aperture tracking with systematic and random multipole errors based on the measurements of the PEP-II magnets. We have used LEGO[7] in the tracking study.

betatron phase apart, during the injection.

The injection aperture should be able to include at least a 6 sigma full width injected beam plus the effective septum width and a 4 sigma half width stored beam. The width of the effective septum is determined by a $1 \mathrm{~mm}$ physical thickness of 


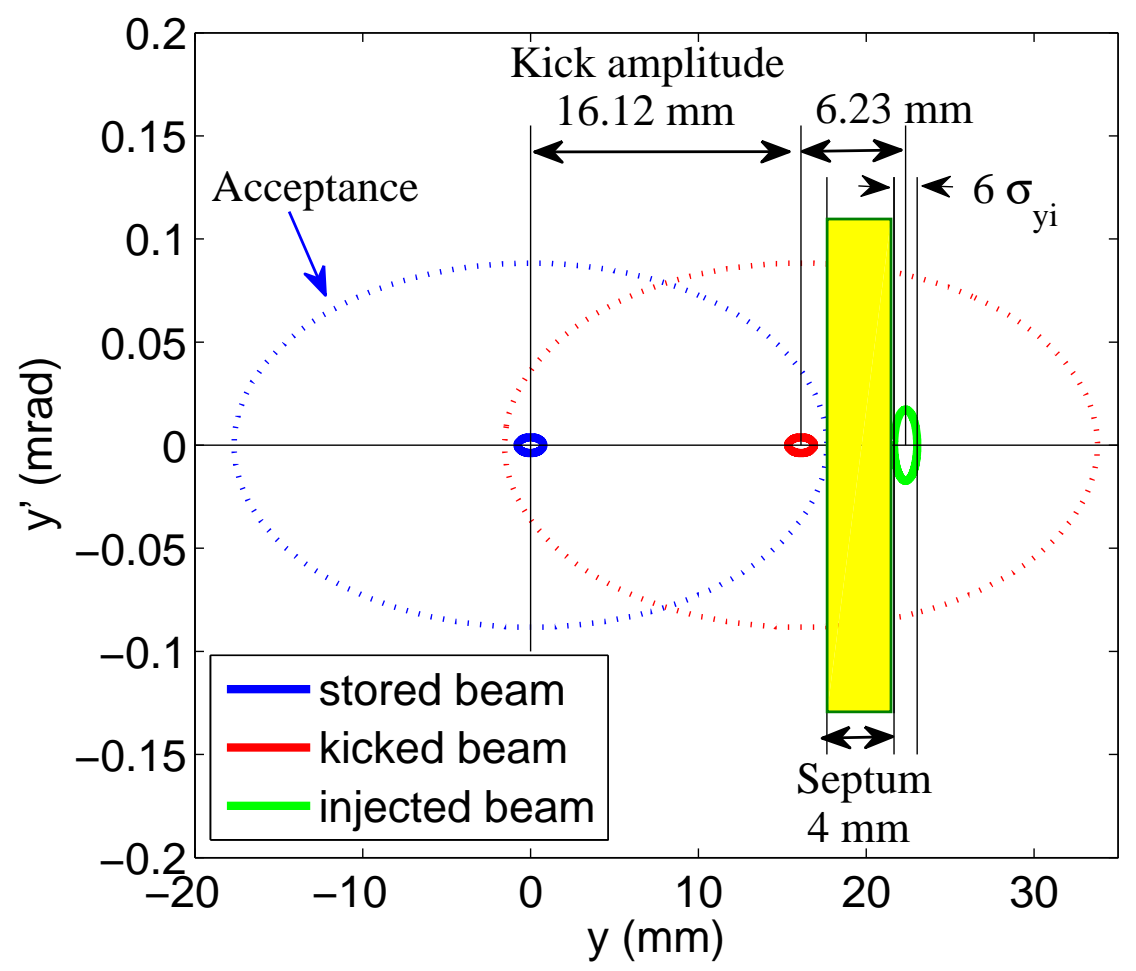

FIG. 17: Injection scheme.

septum plus an allowance of stray field on either side, $1 \mathrm{~mm}$ on the injected beam side and $2 \mathrm{~mm}$ on the stored beam side. The effective septum width is $4 \mathrm{~mm}$. The energy of the injected beam is $4.5 \mathrm{GeV}$ from the linac. The horizontal beam emittance is $5.2 \mathrm{~nm}$-rad which is deduced from the operation of of PEP II. The vertical beam emittance is $1.3 \mathrm{~nm}$-rad which is a quarter of the horizontal beam emittance. The vertical beta function of the injected beam is $40 \mathrm{~m}$ which is optimized to include the 3 sigma injected beam into the acceptance of the storage ring at the injection point as shown in Fig. 17. A reasonably large beta function of the injection plane is 
required to let the septum appear to be thin relative to the aperture. The vertical beta function is $200 \mathrm{~m}$ under these considerations. The full coupling vertical beam emittance without the damping wiggler is used to calculate the stored beam size. The phase space diagram at the injection point is shown in Fig. 17. In the figure the stored beam center is on the closed orbit of a DC bump. In this scheme the stored beam is kicked as close to the septum as possible. This means that the betatron amplitude of injected beam is the smallest after injected into the ring. The kick amplitude is $16.12 \mathrm{~mm}$ and the being injected beam betatron amplitude is $6.23 \mathrm{~mm}$.

\section{STABILITY REQUIREMENTS}

The demanding beam stability requirements for synchrotron light sources include maintaining sufficient constancy in photon beam position, angle, size, energy, and, in some cases, photon pulse time-of-arrival, for users to achieve the spatial and spectral resolution needed for their experiments. Typical specifications for beam pointing stability are $10 \%$ of photon beam's transverse dimensions, while the longitudinal phase stability requirement may be a small fraction of $1^{\circ}$ in order to meet the energy resolution needs $\left(<5 \times 10^{-5}\right)$ or time-of-arrival jitter needs $(<1 \mathrm{ps})$ of demanding experiments. Stability requirements are a function of bandwidth, dependent on experiment data integration times, and component specifications must reflect this. Transverse stability requirements may be modified depending on 1) whether the beam is focused or not; 2) the size of limiting apertures upstream of the experiment (including very small slits or collimators); 3) the presence and nature of diffracting or other optical components; 4) whether acquired data are normalized to the instantaneous incident beam intensity. For example, energy-dependent sam- 
ple absorption measurements may require $<0.1 \%$ noise in the data to resolve fine structure in the spectral scans. Without proper intensity normalization, meeting this noise criterion would require a more demanding pointing stability requirements of $<5 \%$ of the beam dimensions, and a transverse beam size stability of $<0.1 \%$, averaged over the data point acquisition period. In another example, imperfections in a vertically focusing mirror may make it impossible to produce the few-micron vertical spot size of a perfect 1-to-1 imaging system, relaxing the need to stabilize beam position at the source to a small fraction of a micron.

Nevertheless, given the very small photon beam size and divergence of a typical undulator source in PEP-X, extraordinary measures will need to be taken in both the accelerator and beam lines to achieve beam pointing and intensity stability, especially in the vertical plane. These measures include:

- stable design of experimental floor and building

- stable support of magnets and vacuum chambers with nm-level vibration amplitudes and sub-micron-level diurnal stability

- temperature stability on the order of $0.1^{\circ} \mathrm{C}$ for critical accelerator and beam line components

- highly stable (order $10 \mathrm{ppm}$ ) and very low ripple main magnet power supplies

- very high performance beam position monitor (BPM) and orbit feedback systems (beyond the present state of the art) using ultra-stable electron "user BPMs" flanking each insertion device photon source and x-ray BPMs in the beam line 
- optical component feedback systems and photon beam intensity monitors in the beam lines to maintain pointing stability and to normalize acquired data to incident intensity

Achieving these stringent requirements will require an integrated effort from the accelerator and beam line designers to maintain stability integrity in all aspects of hardware and control system design. It may be necessary to implement highresolution ( $100 \mathrm{~nm}$ ) mechanical motion/position survey sensors for critical components in the accelerator (e.g. user BPMs) and beam line (e.g. optical components, small apertures and collimators, etc). Some of these devices may require cuttingedge technology (e.g. "telescope technology" such as the laser-Doppler stabilization system used for atomic force microscopes and the X-ray Nanoprobe at the APS).

Besides mechanical and electrical stability of the magnets, BPM and beam line components, there are a number of high frequency effects which may drive not only transverse but longitudinal bunch instability. These included RF phase and amplitude noise, RF cavity and vacuum chamber impedances, and coherent synchrotron radiation impedances. Mitigations for these effects include high performance lowlevel RF controls, longitudinal and transverse multibunch feedback systems and low impedance vacuum chamber designs. While it is expected that a third harmonic bunch lengthening cavity will provide Landau damping for coupled oscillations, the bunch lengthened mode may complicate fast RF feedback performance, requiring further RF controls development. 


\section{INTRA-BEAM SCATTERING AND TOUSCHEK LIFETIME}

Intra-beam scattering (IBS) describes multiple Coulomb scattering that in electron machines leads to an increase in all bunch dimensions and in energy spread, whereas the Touschek effect concerns large single Coulomb scattering events where energy transfer from transverse to longitudinal leads to immediate particle loss. In low emittance machines, such as PEP-X, both effects tend to be important.

For PEP-X IBS calculations we employ the so-called "high energy approximation" [8], a model that has been shown to give reasonably good agreement in its regime of applicability with the more detailed Bjorken-Mtingwa formulation [9]. We assume that we are coupling dominated, by which we imply that the vertical dispersion can be kept sufficiently small. Then the vertical emittance is proportional to the horizontal emittance,

$$
\epsilon_{y}=\kappa \epsilon_{x}
$$

with $\kappa$ the coupling constant. The nominal (no IBS) horizontal and vertical emittances are given by $\epsilon_{x 0}=\epsilon_{x 00} /(1+\kappa)$ and $\epsilon_{y 0}=\kappa \epsilon_{x 00} /(1+\kappa)$. IBS calculations of the steady-state horizontal emittance $\epsilon_{x}$ and (relative) energy spread $\sigma_{p}$ are performed by simultaneously solving

$$
\epsilon_{x}=\frac{\epsilon_{x 0}}{1-\tau_{x} / T_{x}} \quad \text { and } \quad \sigma_{p}^{2}=\frac{\sigma_{p 0}^{2}}{1-\tau_{p} / T_{p}},
$$

where $\tau_{x}, \tau_{p}$, signify the radiation damping times and $1 / T_{x}, 1 / T_{p}$, the IBS growth rates, and $\sigma_{p 0}$ gives the nominal rms energy spread. Note that the growth rates also depend on the beam emittances and energy spread, and thus the steady-state values of these quantities are on the right hand side of the equations. 
The IBS growth rate in energy spread, according to the high energy approximation, is given by

$$
\frac{1}{T_{p}} \approx \frac{r_{0}^{2} c N(\log )}{16 \gamma^{3} \epsilon_{x}^{3 / 4} \epsilon_{y}^{3 / 4} \sigma_{z} \sigma_{p}^{3}}\left\langle\sigma_{H} g(a / b)\left(\beta_{x} \beta_{y}\right)^{-1 / 4}\right\rangle .
$$

Here $r_{0}$ is the radius of the electron, $c$ the speed of light, $N$ the number of electrons per bunch, $(\log )$ the Coulomb log factor, $\gamma$ the Lorentz energy factor, $\sigma_{z}$ the bunch length, $\beta_{x}, \beta_{y}$, the optical beta functions, and \langle\rangle means to average over the ring. Other factors in Eq. (5) are defined by

$$
\begin{gathered}
\frac{1}{\sigma_{H}^{2}}=\frac{1}{\sigma_{p}^{2}}+\frac{\mathcal{H}_{x}}{\epsilon_{x}}, \quad a=\frac{\sigma_{H}}{\gamma} \sqrt{\frac{\beta_{x}}{\epsilon_{x}}}, \quad b=\frac{\sigma_{H}}{\gamma} \sqrt{\frac{\beta_{y}}{\epsilon_{y}}} \\
g(\alpha)=\alpha^{(0.021-0.044 \ln \alpha)}
\end{gathered}
$$

where $\mathcal{H}$ is the so-called "curly $\mathrm{H}$ " dispersion function. Finally, the horizontal IBS growth rate is given by

$$
\frac{1}{T_{x}}=\frac{\sigma_{p}^{2}\left\langle\mathcal{H}_{x}\right\rangle}{\epsilon_{x}} \frac{1}{T_{p}}
$$

The high energy IBS approximation given here has validity when $a, b \ll 1$, which holds for the PEP-X parameters.

In scattering calculations, like IBS, a Coulomb log term is used to take into account the contribution of very large and very small impact parameter events. Due to the very small impact parameter events, the tails of the steady-state bunch distributions are not Gaussian and the standard way of computing (log) overemphasizes their importance. To better describe the size of the core of the bunch we calculate the Coulomb log factor as first proposed by Raubenheimer [10],[11]. For PEP-X, $(\log ) \approx 10$. 
For our IBS calculations nominal parameters are obtained from Table I, and the lattice used is that described earlier. We assume 3440 bunches in the ring; we consider as nominal bunch lengths $\sigma_{z 0}=2.5 \mathrm{~mm}$ and $\sigma_{z 0}=5.0 \mathrm{~mm}$, which we believe can be reached with the use of a higher harmonic cavity. We assume that potential well bunch lengthening is not significant and that the nominal current is below the threshold of the microwave instability. Results, for steady-state emittances $\epsilon_{x}, \epsilon_{y}$, as functions of beam current $I$ are shown in Figs. 18 and 19. Results are given for $\kappa=0.03$ (blue), 0.10 (red), 0.30 (yellow), 1 (green), when $\sigma_{z}=2.5$, and results for $\sigma_{z}=5.0 \mathrm{~mm}$ are indicated by the dotted curves.

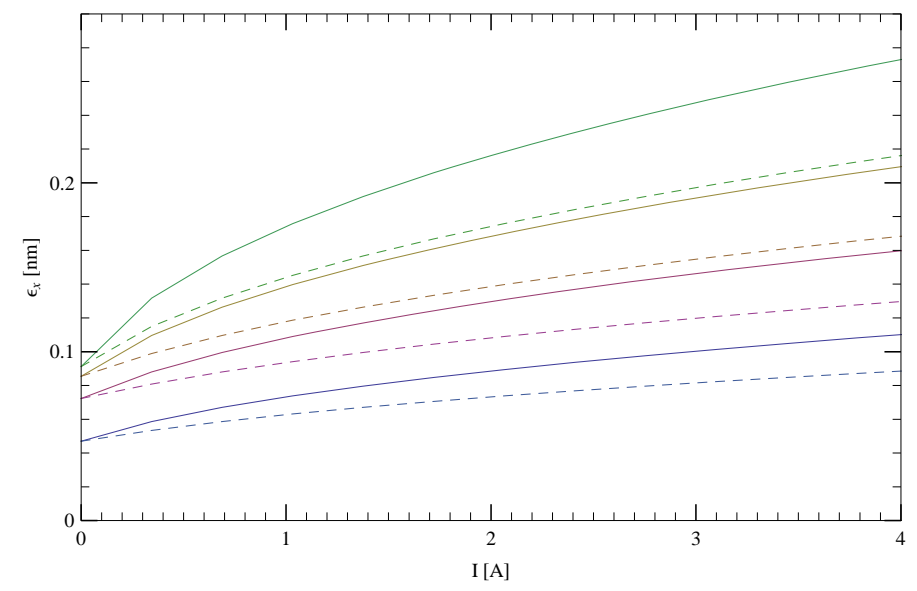

FIG. 18: Steady-state horizontal emittance as a function of bunch current for $\kappa=0.03$ (blue), 0.10 (red), 0.30 (yellow), 1 (green), when $\sigma_{z}=2.5 \mathrm{~mm}$. The results for $\sigma_{z}=5.0 \mathrm{~mm}$ are given by the dotted curves.

For PEP-X, $\sigma_{p}$ and $\sigma_{z}$ grow little with current: for $I=1.5 \mathrm{~A}, \sigma_{z}=2.5 \mathrm{~mm}$, and $\kappa=3 \%$ they grow by only $6 \%$. This is true because the quantity $\left(\tau_{x} \sigma_{p}^{2}\left\langle\mathcal{H}_{x}\right\rangle / \tau_{p} \epsilon_{x}\right)$ 


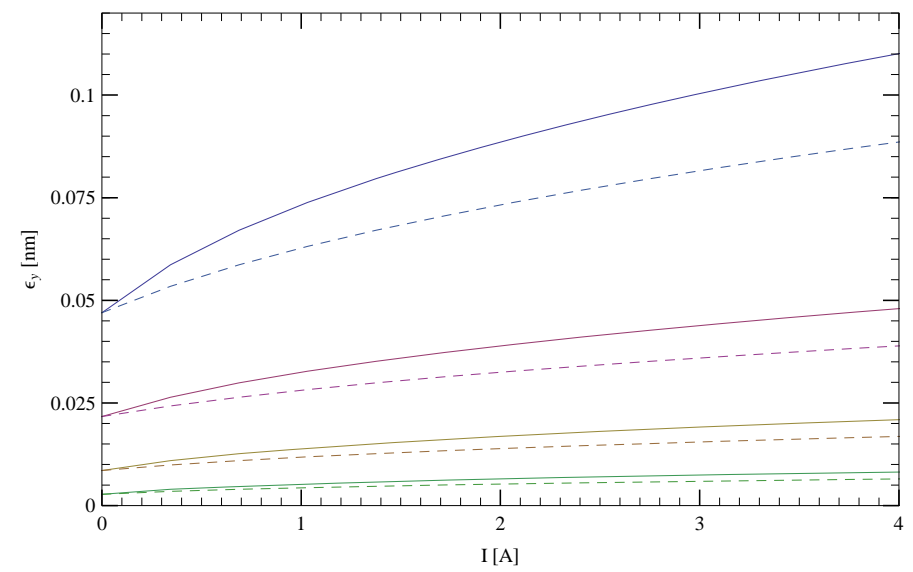

FIG. 19: Steady-state vertical emittance as a function of bunch current for $\kappa=0.03$ (blue), 0.10 (red), 0.30 (yellow), 1 (green), when $\sigma_{z}=2.5 \mathrm{~mm}$. The results for $\sigma_{z}=5.0 \mathrm{~mm}$ are given by the dotted curves.

happens to be large (see Eq. 8), with a nominal value of 9 . This means that, to good approximation, $\sigma_{p}$ and $\sigma_{z}$ can be taken to have their nominal values and one need only solve the first of Eqs. (4). In fact, the emittance as a function of peak current can be obtained from the solution of a single equation of the form $\left(\epsilon_{x} / \epsilon_{x 0}\right)^{5 / 2}-\left(\epsilon_{x} / \epsilon_{x 0}\right)^{3 / 2}-\alpha N / \sigma_{z}=0$, with $\alpha$ a constant. To allow one to reproduce the curves of Figs. 18 and 19 from this equation, note that the coupling depedence of $\alpha$ can be taken to be $(1+\kappa)^{5 / 2} \kappa^{-3 / 4+0.011(1-\ln \kappa)}$.

In Table IV we give steady-state emittances for $I=1.5 \mathrm{~A}$ for the two nominal bunch lengths and for two couplings. At full coupling $\kappa=1$, the horizontal emittance $\left(\epsilon_{x}, \epsilon_{y}\right)$ is minimized. The other couplings were chosen to give diffraction limited $\epsilon_{y}$ at 1 angstrom wavelength, $\epsilon_{y}=8 \mathrm{pm}$. Note that, due to IBS, we cannot quite reach 
the diffraction limited $\epsilon_{y}$ and at the same time have $\epsilon_{x}=0.1 \mathrm{~nm}$.

TABLE IV: Steady-state emittance and Touschek lifetime at $I=1.5$ A for the two nominal values of bunch length $\sigma_{z}$. In each case a full coupling result $\kappa=1$ and one which yields $\epsilon_{y}=8 \mathrm{pm}$ are given.

\begin{tabular}{|c|c|c|c|c|}
\hline$\sigma_{z}[\mathrm{~mm}]$ & $\kappa$ & $\epsilon_{x}[\mathrm{~nm}-\mathrm{rad}]$ & $\epsilon_{y}[\mathrm{~nm}-\mathrm{rad}]$ & $T_{l}[\mathrm{~min}]$ \\
\hline 2.5 & 1 & 0.082 & 0.082 & 31 \\
\hline 2.5 & 0.045 & 0.18 & 0.0082 & 10.3 \\
\hline 5.0 & 1 & 0.068 & 0.068 & 57 \\
\hline 5.0 & 0.055 & 0.14 & 0.0079 & 19.4 \\
\hline
\end{tabular}

The Touschek lifetime calculations follow the method of Brück [12],[13]. We take as momentum acceptance $\Delta p / p= \pm 1.5 \%$, which was found by dynamic aperture studies. Touschek lifetimes $T_{l}$ are given in the last column of Table IV. Note that these calculations are based on the IBS determined, steady-state beam sizes (otherwise the results would be much smaller). In the fully coupled cases, $T_{l} \sim$ $1.5-2$ hours; in the diffraction limited cases $T_{l} \sim 0.5$ hour.

Finally, note that since both IBS and the Touschek effect depend on $N$ and $\sigma_{z}$ only as their ratio $N / \sigma_{z}$ (see e.g., Eq. 5 ) the $I=3.0 \mathrm{~A}, \sigma_{z}=5.0 \mathrm{~mm}$, emittances and lifetimes are identical to the ones when $I=1.5 \mathrm{~A}, \sigma_{z}=2.5 \mathrm{~mm}$.

\section{COLLECTIVE EFFECTS}

The impedance of the RF cavities and vacuum chamber can drive single bunch and coupled-bunch instabilities in the ring. Without engineering designs of the cavities and the chamber, it is not possible to make accurate assessments of the 
thresholds and growth rates associated with the instabilities. In the following we will focus on three subjects: microwave instability, coherent synchrotron instability, and multibunch transverse instability due to the resistive wall impedance.

\begin{tabular}{|l|c|}
\hline Circumference, $(\mathrm{m})$ & 2199.32 \\
\hline Average $I_{\text {beam }},(\mathrm{A})$ & 3 \\
\hline Number of bunches, $N_{b}$ & 3388 \\
\hline Peak current, $(\mathrm{A})$ & 414 \\
\hline$\nu_{x}$ & 86.23 \\
\hline$\nu_{y}$ & 36.14 \\
\hline$\nu_{s}$ & 0.00742 \\
\hline$\alpha$ & $4.72 \times 10^{-5}$ \\
\hline$\sigma_{z},(\mathrm{~mm})$ & 2.5 \\
\hline$\sigma_{\delta}$ & $1.12 \times 10^{-3}$ \\
\hline Damp. time, long., $\tau_{l},(\mathrm{~ms})$ & 10 \\
\hline Length of arcs, $(\mathrm{m})$ & 1522.4 \\
\hline Length of insertions and wigglers, $(\mathrm{m})$ & 180 \\
\hline Length Straights, $(\mathrm{m})$ & 337 \\
\hline
\end{tabular}

TABLE V: Parameters of the ring.

We do not consider in this preliminary study multibunch instabilities driven by higher order modes in RF cavities. Such analysis requires knowledge of the frequencies and shunt impedances of HOMs, which are not available at this time. Note however, that these instabilities are insensitive to the bunch length. Based on the experience of the Low Energy Ring in PEP-II (with the maximal total current of $3 \mathrm{~A}$, and the beam energy of $3.1 \mathrm{GeV}$ ) we expect that these instabilities can be stabilized with transverse and longitudinal feedbacks. 
We will also skip analysis of transverse mode coupling instability (TMCI) of the beam. This instability is typically less dangerous than the longitudinal microwave.

We use some plausible assumptions regarding the impedance of the machine. We should emphasize that our results are for a preliminary and still largely incomplete model of the machine impedance and meaningful conclusions should wait until a more comprehensive impedance model is developed. We use the parameters in Table $\mathrm{V}$ for the ring.

\section{A. Microwave instability}

To study microwave instability of the beam, a short range wakefield is required. In what follows, we will estimate the threshold for the instability using the wake calculated for the Low Energy Ring of PEP-II $[14,15]$. This wake includes short range contributions from the beam position monitors, RF cavities, resistive wall impedance, as well as some other elements of the ring. To smooth out the singularity of the wake at the origin (due to the accepted models for the resistive wall and the inductive wakefields, see [15]), this wake was convoluted with a Gaussian distribution with rms length of $0.5 \mathrm{~mm}$. The plot of the convoluted wake is shown in Fig. 20. The positive value means energy loss, and $s>0$ corresponds to positions behind the source particle.

For stability analysis we used a linearized Vlasov code that calculates the growth rate of the microwave instability for a given current of the beam [16]. The code does not take into account the synchrotron damping time. This means that the growth rates calculated with the code should be compared with the inverse longitudinal

damping time $\tau_{d}^{-1}\left(0.1 \mathrm{~ms}^{-1}\right)$. Only a growth rate that is larger then $\tau_{d}^{-1}$ leads 


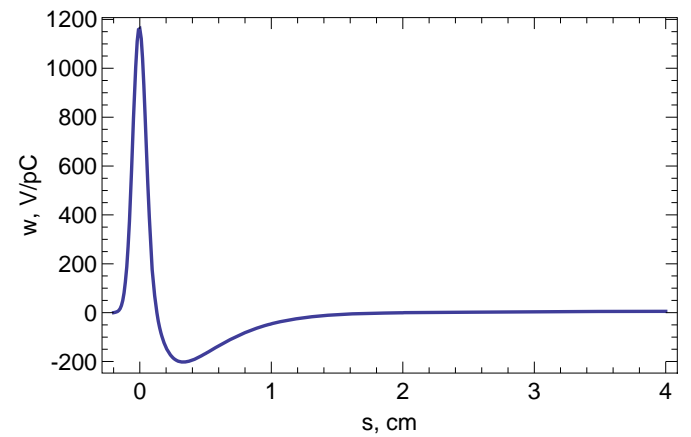

FIG. 20: The LER short range wake (in units of V/pC) convoluted with a $0.5 \mathrm{~mm}$ Gaussian distribution.

to a real instability. Calculations were carried out for two different bunch lengths, $\sigma_{z}=2.5 \mathrm{~mm}$ and $\sigma_{z}=5 \mathrm{~mm}$. The results are shown in Fig. 21. As is seen from this

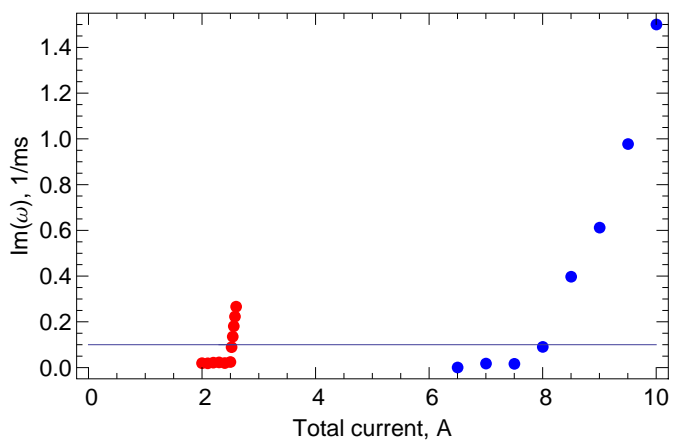

FIG. 21: Thresholds for the microwave instability for $2.5 \mathrm{~mm}$ (red dots) and $5 \mathrm{~mm}$ (blue dots) rms bunch length versus the total current in the ring. The horizontal line gives the value of the inverse synchrotron time $0.1 \mathrm{~ms}^{-1}$.

figure, the threshold for the microwave instability for a $2.5 \mathrm{~mm}$ bunch corresponds to 
the total current $I$ in the ring being equal to $2.5 \mathrm{~A}$; for the $5 \mathrm{~mm}$ bunch it increases to about $8 \mathrm{~A}$. In the calculation of the total current we assumed 3388 bunches in the ring.

It is important to note that the actual bunch length, at the current close the the instability threshold, increases relative to the zero current case. As an example, Fig. 22 shows longitudinal distribution in the bunch (for $2.5 \mathrm{~mm}$ nominal (that

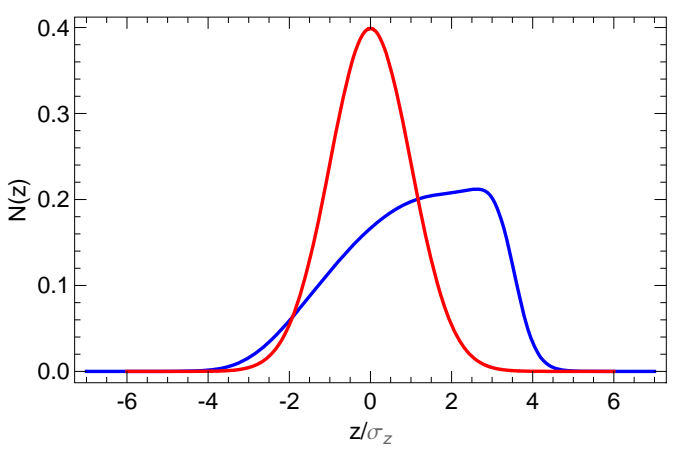

FIG. 22: Longitudinal distribution for a bunch of zero current (red line) and $I=2.5 \mathrm{~A}$ (blue line). Positive values of $z$ correspond to the head of the bunch.

is zero current) rms bunch length) at $I=2.5 \mathrm{~A}$. This equilibrium distribution is obtained by solving the Haissinski equation with the LER wake. One can see that the bunch distribution is tilted in the forward direction; its rms bunch length is approximately equal to $5 \mathrm{~mm}$.

\section{B. Microwave instability due to coherent synchrotron radiation}

The threshold current of the CSR driven instability is typically determined by the wavelengths shorter than the natural bunch length. The CSR instability threshold 
for a coasting beam in free space is [17] is given by the following equation

$$
\frac{\hat{I}_{t h}}{I_{A}}=\frac{\gamma \alpha_{c} \sigma_{\delta}^{2} c T_{0}}{2\left(\pi R \lambda^{2}\right)^{1 / 3}}
$$

where $\hat{I}_{t h}$ is the threshold peak current, and $R$ is the dipole bending radius. Note that the threshold current is proportional to the energy spread squared, and does not depend on the bunch length. It is determined by the longest wavelength before vacuum chamber shielding takes effect. Using a parallel plate model, the shielding cutoff wavelength can be determined as [18]

$$
\lambda_{s}=\frac{2^{5 / 2} g^{3 / 2}}{\sqrt{\pi} R^{1 / 2}},
$$

where $g$ is the half height of the vacuum chamber. Taking $g=2 \mathrm{~cm}$ for a typical vacuum chamber, we find that the shielding cut-off wavelength is about $0.9 \mathrm{~mm}$.

Using $\lambda=\lambda_{s}$ in Eq. (9), we can estimate the threshold peak current as

$$
\frac{\hat{I}_{t h}}{I_{A}}=\frac{\gamma \alpha_{c} \sigma_{\delta}^{2} c T_{0}}{2^{8 / 3} g}
$$

With Eq. (11) we estimate that the threshold peak current is 154 A. For Gaussian bunches with rms bunch length of $2.5 \mathrm{~mm}$ this gives the threshold for the total current in the ring to be $1.5 \mathrm{~A}$. More studies on the microbunching instability driven by CSR with realistic vacuum chamber geometry are necessary to determine the threshold current more accurately.

\section{Multibunch transverse instability due to the resistive wall wakefield}

For calculation of the growth rate for the transverse multibunch instability we use the following result from [19] for the frequency shift induced by the long range 
wakefields:

$$
\Delta \omega(l)=-i \frac{M N r_{e} c}{2 \gamma T_{0}^{2} \omega_{\beta}} \sum_{p=-\infty}^{\infty} Z_{t}\left[\omega_{\beta}+(p M+l) \omega_{0}\right],
$$

where $Z_{t}(\omega)$ is the transverse impedance, $M$ is the number of bunches in the ring, $N$ is the number of particles in the bunch, $r_{e}$ is the electron classical radius, $\gamma$ is the relativistic factor, $T_{0}$ is the revolution period in the ring, $l$ is an integer number of the mode, and $\omega_{\beta}$ is the betatron frequency. The formula assumes a uniform distribution of bunches in the ring and treats bunches as point charges with the charge equal to $N e$.

We can carry out the summation analytically, if we use wakefields instead of impedances. In terms of wakefield, Eq. (12) can be written as follows

$$
\Delta \omega(l)=\frac{N r_{e} c}{2 \gamma T_{0} \omega_{\beta}} \sum_{n=1}^{\infty} w_{t}\left(n s_{b}\right) e^{2 \pi i\left(l+\nu_{\beta}\right) n / N_{b}} .
$$

For the resistive wall the transverse wake decays with distance as $w_{t}=A z^{-1 / 2}$ and the sum can be computed analytically in terms of the polylogarithm function $\operatorname{Li}_{\frac{1}{2}}(x)$ : $\operatorname{Li}_{k}(x)=\sum_{n=1}^{\infty}\left(x^{n} / n^{k}\right)$, so that

$$
\Delta \omega(l)=\frac{N r_{e} c}{2 \gamma T_{0} \omega_{\beta}} w_{t}\left(s_{b}\right) \operatorname{Li}_{\frac{1}{2}}\left(e^{2 \pi i\left(l+\nu_{\beta}\right) / M}\right) .
$$

The function $\operatorname{Li}_{\frac{1}{2}}(x)$ is a periodic function with the period equal to 1. Its imaginary part diverges, $\operatorname{Li}_{\frac{1}{2}}(x) \rightarrow+\infty$, when $x+\rightarrow 0$, which means that the maximum growth rate is attained for the minimal value of the argument $\left(l+\nu_{\beta}\right) / M$. This value is equal to $-\left(1-\left[\nu_{\beta}\right]\right) / M$ where $\left[\nu_{\beta}\right]$ is the fractional part of the tune. For small negative values of the argument $x$, the function $\operatorname{Li}_{\frac{1}{2}}\left(e^{2 \pi i x}\right)$ can be approximated by $(1-i) / 2 \sqrt{-x}$ which gives the following equation for the approximate value of 
the growth rate of the instability

$$
\operatorname{Im} \Delta \omega(l)=\frac{N r_{e} c}{4 \gamma T_{0} \omega_{\beta}} w_{t}\left(s_{b}\right) \sqrt{\frac{M}{1-\left[\nu_{\beta}\right]}} .
$$

Applying this formula for the transverse resistive wall wake we will use the following expression for the transverse resistive wall wake

$$
w_{t}(z)=\frac{A}{\sqrt{z}} .
$$

Using this expression for the wake and taking into account that $s_{b}=C / M$, we can rewrite Eq. (15) for the growth rate of the instability as follows

$$
\operatorname{Im} \Delta \omega=\frac{A c^{2}\left(I_{\mathrm{av}} / I_{\mathrm{A}}\right)}{4 \gamma \omega_{\beta} \sqrt{C\left(1-\left[\nu_{\beta}\right]\right)}} .
$$

The factor $A$ (in CGS units) is given by

$$
A=\frac{2 \sqrt{c}}{\pi} \sum_{i} \frac{L_{i}}{b_{i}^{3} \sigma_{c i}}
$$

where the subscript $i$ indicates various regions of the ring (arcs, straights, insertions, and the wiggler), $L_{i}$ is the length of the region $i, b_{i}$ is the pipe radius, and $\sigma_{c i}$ is the wall conductivity of the vacuum chamber in region $i$. In this calculation we assume a round cross section for the vacuum chamber. We used dimensions $b_{i}$ for the vacuum chamber shown in Table VI: We assume aluminum (resistivity

\begin{tabular}{|c|c|c|c|}
\hline Arc & Straight & Insertions & Wiggler \\
\hline 2.8 & 4.8 & 0.3 & 0.75 \\
\hline
\end{tabular}

TABLE VI: Radius $b_{i}$ (in $\mathrm{cm}$ ) of the vacuum chamber in various regions of the ring.

26.0 $10^{-7} \mathrm{Ohm}$ Centimeter) for the arcs and straights, and copper for the wiggler and insertions (resistivity $17.7 \cdot 10^{-7} \mathrm{Ohm}$ Centimeter). This gives $A=505 \mathrm{~V} /(\mathrm{pC} \sqrt{\mathrm{m}})$, and the growth rate of the instability $0.19 \mathrm{~ms}$ (corresponding approximately to 25 revolutions). 


\section{FAST ION INSTABILITY}

Ions generated by beam-gas ionization can be trapped by the electron bunches. The ion-cloud can cause the beam instability, emittance blow-up and tune shift. The ion induced beam instability is one critical issue for PEP-X due to its ultra small emittance.

The exponential growth rate of fast ion instability is given by [20]

$$
\frac{1}{\tau_{c, y}}=\frac{c r_{e} \beta_{y} N n_{b}}{2} \frac{\hat{W}}{\gamma} .
$$

Where $\hat{W}$ is the coupling force between the electron-bunches and ions

$$
\hat{W}=\frac{8 \sigma_{i} P}{3 \sqrt{3} k T}\left(\frac{r_{p}}{A}\right)^{1 / 2} \frac{\left(N S_{b}\right)^{1 / 2} n_{b}}{\sigma_{y}^{3 / 2}\left(\sigma_{y}+\sigma_{x}\right)^{3 / 2}} .
$$

Where $P$ is the pressure, $\sigma_{i}$ is the ionization cross-section, $A$ is mass number of ion, $r_{p}$ is the classical radius of proton, $k$ is Boltzmann's constant, $\mathrm{T}$ is the temperature, $n_{b}$ is the number of bunches, $N$ is the number of electrons per bunch, $\sigma_{x, y}$ is the transverse beam size and $S_{b}$ is the bunch spacing. The coupling force in PEP$\mathrm{X}$ is about 3 orders of magnitude larger than that in B-factories due to its small emittance.

On the other hand, the ultra small beam size can mitigate the instability by driving the ion unstable and providing more effective landau damping. Without gaps in the beam fill pattern, the ions with a relative molecular mass greater than $A_{x, y}$ will be trapped horizontally (vertically), where

$$
A_{x, y}=\frac{N r_{p} S_{b}}{3\left(\sigma_{x}+\sigma_{y}\right) \sigma_{x, y}}
$$

If the beam size is small enough, the strong beam's force can overly focus the ions and causes the ion's motion unstable. Fig. 23 shows the critical mass number $A_{x, y}$ 
along a quarter of the ring for $10 \%$ beam coupling. The $\mathrm{H}_{2}^{+}, \mathrm{CH}_{4}^{+}, \mathrm{H}_{2} \mathrm{O}^{+}$are unstable in most of the regions and $\mathrm{CO}^{+} / \mathrm{N}_{2}^{+}$ions are unstable at partial regions as shown in the figure. According to Eq.(21), there are less number of ions trapped with a smaller coupling.

One important damping mechanism is the ion oscillation frequency spread $\Delta \omega_{i}$ along the ring due to the variation of beam size [21]

$$
\frac{1}{\tau_{e}} \approx \frac{1}{\tau_{c}} \frac{c}{2 \sqrt{2} n_{b} S_{b} \Delta \omega_{i}}
$$

The oscillation frequency of the trapped ions is given by

$$
\omega_{i, x(y)}=\left(\frac{4 N r_{p} c^{2}}{3 A S_{b}\left(\sigma_{x}+\sigma_{y}\right) \sigma_{x, y}}\right)^{1 / 2}
$$

The large frequency spread of $150 \mathrm{MHz}$, as shown in Fig. 24, provides a significant landau damping of the beam instability. The DBA sections have a larger spread than the TME sections.

A gap between bunch trains can be added to suppress the ion trapping. Our study shows that the ion density exponentially decays during a train gap. With a multi-train beam filling pattern, the ion density can be reduce by a factor of $F_{\text {train }}[22]$

$$
F_{\text {train }}=\frac{1}{N_{\text {train }}} \frac{1}{1-\exp \left(-\tau_{\text {gap }} / \tau_{\text {ions }}\right)}
$$

Here, $\tau_{\text {ions }}$ is the diffusion time of the ion-cloud, which is close to the ion oscillation period. Also, $\tau_{\text {gap }}$ is the length of bunch train gaps and $N_{\text {train }}$ is the number of bunch trains.

The beam instability is simulated with a strong-weak code. Each bunch is represented by one macro-bunch, but the ions are represented by many macro-particles. 
The electron bunches interact with the ions at each element when they are passing by. Therefore, the effects of trapping condition, train gap and Landau damping are all included in the simulation. Different beam filling patterns are investigated. The assumed residual gas molecular species in the vacuum chamber are shown in Table VII. We assume a constant pressure of 1 nTorr along the whole ring. The simulated growth time is shown in Table VIII. There is a faster instability in the full coupling case since more ions can be trapped. With 8 bunch trains, there is a similar vertical growth time of $50 \mu$ s for $100 \%, 10 \%$ and $5 \%$ coupling due to the balanced effects of the coupling force, trapping condition and landau damping discussed above.

The feedback time of the present PEP-II feedback system is $500 \mu \mathrm{s}$. The growth time of $50 \mu$ s with nominal bunch number 3440 is 10 times faster than the feedback. A good vacuum of $0.1 \mathrm{nTorr}$ is required for a $500 \mu \mathrm{s}$ of FII growth time if the beam filling pattern of $8 \times 430$ is chosen. A way of compromising is to reduce the number of bunches to $3237(83 \times 39)$. This bunch-train filling pattern can significantly reduce the number of trapped ions and a growth time of $300 \mu$ s with 1 nTorr is achievable. In this case, a pressure of 0.5 nTorr would meet the feedback requirement.

\begin{tabular}{|c|c|c|c|}
\hline & Cross section (Mbarn) & Mass number & Percentage \\
\hline $\mathrm{H}_{2}$ & 0.35 & 2 & $75 \%$ \\
\hline $\mathrm{CO}$ & 2.0 & 28 & $14 \%$ \\
\hline $\mathrm{CO}_{2}$ & 2.92 & 44 & $7 \%$ \\
\hline $\mathrm{CH}_{4}$ & 2.1 & 16 & $4 \%$ \\
\hline
\end{tabular}

TABLE VII: Parameters of the main molecular species in the vacuum 


\begin{tabular}{|c|c|c|c|c|}
\hline Coupling & Number of bunches & Beam filling pattern & $\tau_{x}(\mu \mathrm{s})$ & $\tau_{y}(\mu \mathrm{s})$ \\
\hline $100 \%$ & 3440 & $1 \times 3440$ & 42 & 12 \\
\hline \hline & 3440 & $8 \times 430$ & 105 & 40 \\
\hline \hline $10 \%$ & 3440 & $1 \times 3440$ & 112 & 18 \\
\hline \hline & 3440 & $8 \times 430$ & 130 & 50 \\
\hline \hline & 3237 & $83 \times 39$ & 3300 & 294 \\
\hline \hline $5 \%$ & 2988 & $83 \times 36$ & 3400 & 394 \\
\hline \hline & 3440 & $1 \times 3440$ & 116 & 24 \\
\hline
\end{tabular}

TABLE VIII: Simulated beam growth rate with different coupling and beam filling patterns (bunch-train number $\times$ number of bunch per train). The total vacuum pressure is $1 \mathrm{nTorr}$ and the total beam current is $3 \mathrm{~A}$.

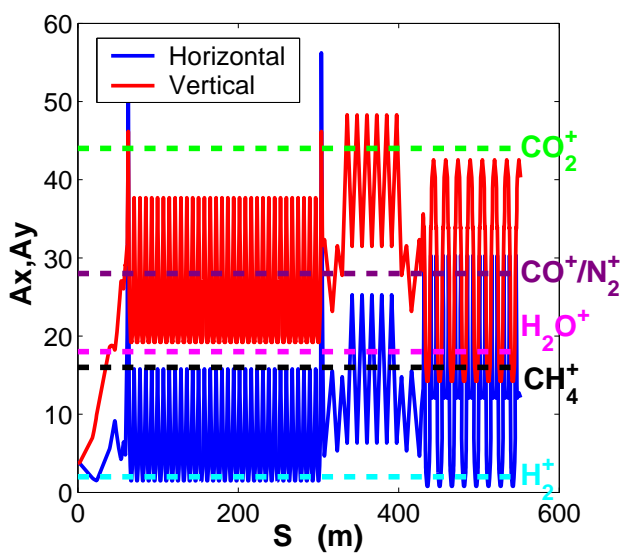

FIG. 23: Critical mass number for $10 \%$ coupling. The mass number of $\mathrm{H}_{2}, \mathrm{CH}_{4}, \mathrm{H}_{2} \mathrm{O}, \mathrm{CO}$ and $\mathrm{CO} 2$ is marked in the plot. 


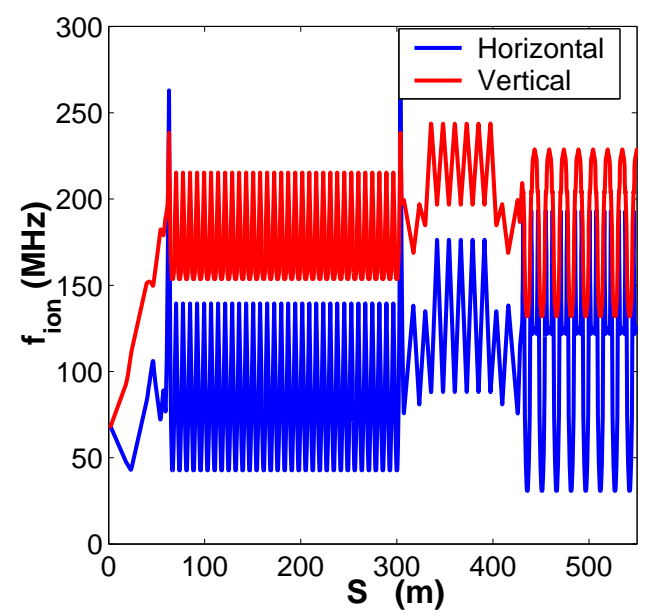

FIG. 24: The $\mathrm{CO}^{+}$frequency along quarter of the ring, $10 \%$ coupling

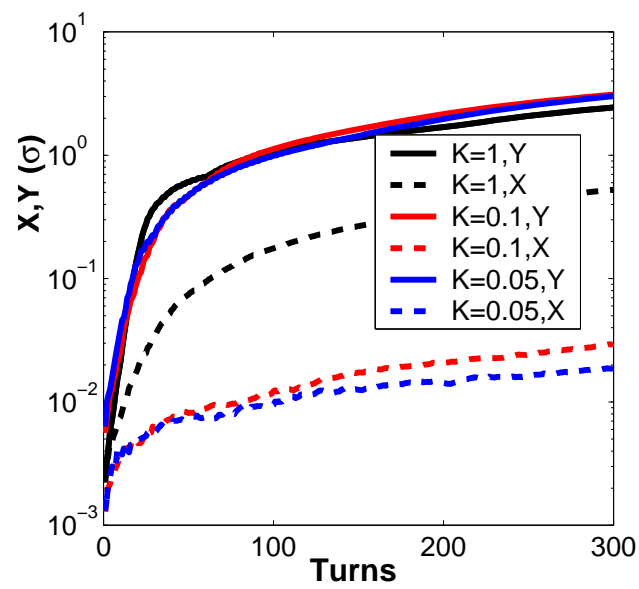

FIG. 25: Simulated bunch amplitude as a function of time for different couplings. The beam consists of 8 bunch-trains. The amplitude is normalized by the beam size 


\section{LATTICE MIGRATION FROM PEP-II TO PEP-X}

The PEP-II upgrade to PEP-X can be performed in several stages, where only part of the machine is upgraded at each stage. This approach would allow to spreading the cost of the complete upgrade over a longer time and allowing earlier operation and testing of the upgraded lattice and beamlines. The proposed migration scenario is shown in Fig. 26. The main upgrades at each stage are as follows:

1. PEP-II:

- The existing machine is setup for $4.5 \mathrm{GeV}$ beam operation, and the FODO cell phase advance in four arcs $5,7,9,11$ is increased from $60^{\circ}$ to $90^{\circ}$ for a lower emittance. The other two arcs remain at $60^{\circ}$ phase advance to preserve conditions at the local sextupoles for compensation of the Interaction Region (IR) chromaticity.

2. PEP-2.1:

- The IR low $\beta$ section is replaced with a FODO straight section.

- Phase advance in the $\operatorname{arcs} 1,3$ is increased from $60^{\circ}$ to $90^{\circ}$. Special IR bending adjustment in four arc dipoles is removed and the affected magnet positions near the straight sections are adjusted. 40 additional sextupoles are installed in these arcs.

- A $90 \mathrm{~m}$ wiggler is installed in straight sections 2 and 6 .

3. PEP-2.2:

- FODO lattice in $\operatorname{arcs} 1,7$ is replaced with the DBA lattice.

- Photon beamlines are installed. 
- The present RF cavities in straight 12 are relocated to straight 4 to free space for an additional beamline.

4. PEP-X:

- FODO lattice in $\operatorname{arcs} 3,5,9,11$ is replaced with the TME lattice.

- A few quadrupole positions in straight sections are adjusted for a better match to the TME arcs.

- Injection system is adjusted for maximum injection efficiency.

The intermediate stage PEP-2.1 can be used for testing the damping wiggler performance, operation with low beam emittance and possibly utilize the existing two beamlines in PEP-II which use the HER arc dipoles as ID. The PEP-2.2 stage will provide the DBA lattice for ID installation and initial operation of photon beamlines. The final PEP-X stage will provide the low emittance design for the highest brightness.

The lattice parameters for the proposed migration stages are listed in Table IX. For all stages, the beam energy of $4.5 \mathrm{GeV}, \mathrm{RF}$ voltage of $10 \mathrm{MV}$ and the $90 \mathrm{~m}$ wiggler are assumed. The number of beamlines in the last row includes the existing two HER beamlines which can use dipoles as ID in PEP-II and PEP-2.1 stages, and the two new beamlines built in straights 12 and 6 in PEP-2.2 and PEP-X stages. 

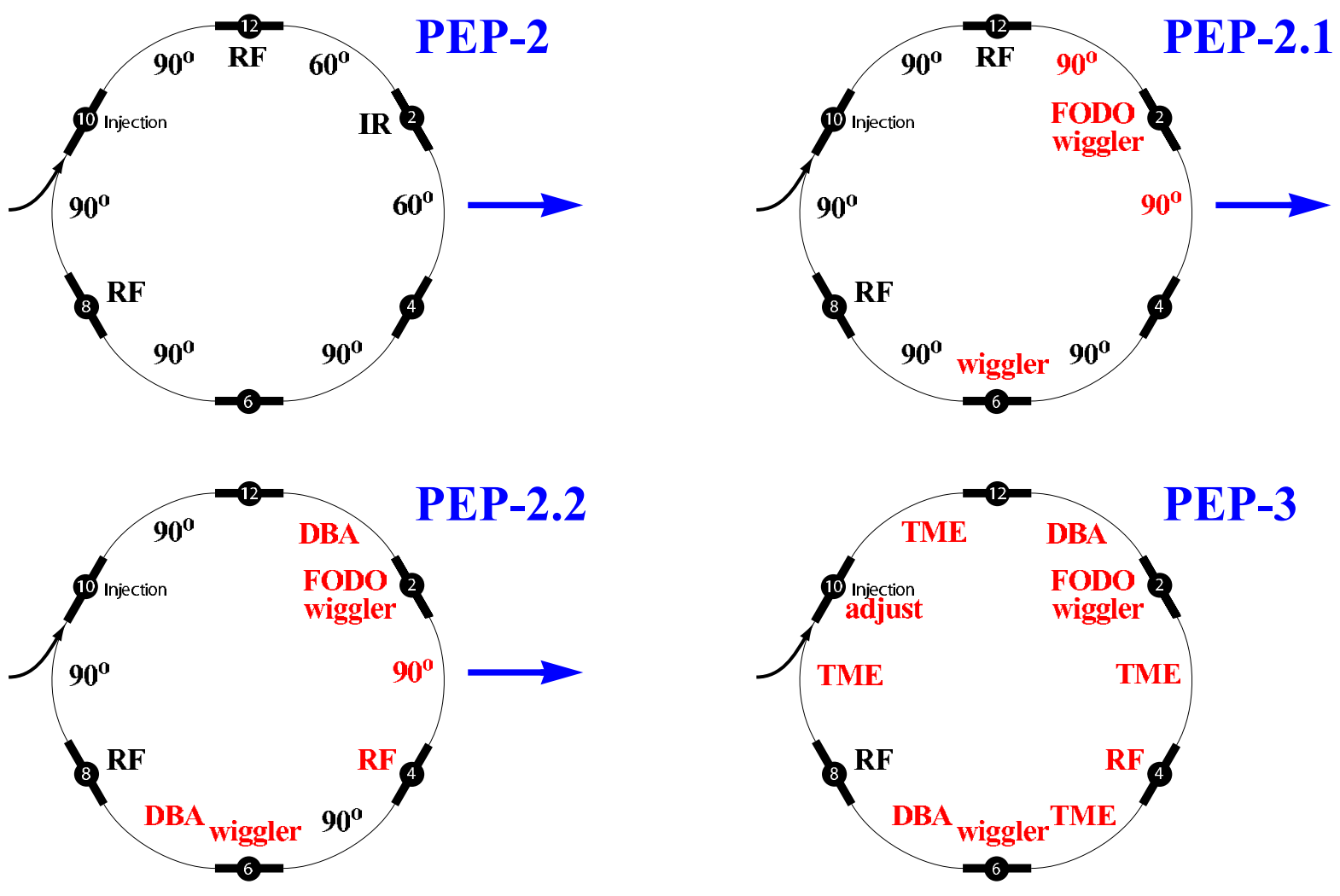

FIG. 26: Lattice migration stages. 
TABLE IX: Parameters for the lattice migration stages.

\begin{tabular}{|l|c|c|c|c|}
\hline Migration optics & PEP-II & PEP-2.1 & PEP-2.2 & PEP-X \\
\hline Betatron tune, $x / y$ & $28.529 / 29.61$ & $31.19 / 32.23$ & $47.105 / 32.13$ & $86.23 / 36.14$ \\
\hline Synchrotron tune & 0.0458 & 0.0398 & 0.0324 & 0.0074 \\
\hline Momentum compaction & $1.69 \cdot 10^{-3}$ & $1.33 \cdot 10^{-3}$ & $8.95 \cdot 10^{-4}$ & $4.72 \cdot 10^{-5}$ \\
\hline Emittance without IBS $[\mathrm{nm}]$ & 7.4 & 0.41 & 0.32 & 0.094 \\
\hline RMS bunch length $[\mathrm{mm}]$ & 3.9 & 13.8 & 11.0 & 2.5 \\
\hline RMS momentum spread & $3.0 \cdot 10^{-4}$ & $1.18 \cdot 10^{-3}$ & $1.14 \cdot 10^{-3}$ & $1.12 \cdot 10^{-3}$ \\
\hline Damping time, $x / y / s[\mathrm{~ms}]$ & $295 / 297 / 151$ & $23 / 23 / 12$ & $20 / 21 / 11$ & $20 / 20 / 10$ \\
\hline Natural chromaticity, $x / y$ & $-55.6 /-72.8$ & $-46.1 /-41.0$ & $-62.3 /-57.9$ & $-132.7 /-72.8$ \\
\hline Energy loss $[\mathrm{MeV} /$ turn] & 0.22 & 2.84 & 3.16 & 3.27 \\
\hline Number of photon beamlines & 2 & 2 & 32 & 32 \\
\hline
\end{tabular}




\section{CONCLUSION}

The selection of topics studied in the report is based on the experience we have accumulated over many years while working on SPEAR3, PEP-II, and ILC damping rings. The study shows that there is no show-stopper and the goals discussed at the beginning of this paper are realistic and achievable.

A summary of the main achievable parameters is shown in Table X.

\begin{tabular}{lll}
\hline \hline Parameter & Description & Value \\
\hline$E(\mathrm{GeV})$ & Beam energy & 4.5 \\
$C(\mathrm{~m})$ & Circumference & 2199.32 \\
$\epsilon_{x}(\mathrm{~nm}-\mathrm{rad})$ & Horizontal emittance & 0.14 \\
$\epsilon_{y}(\mathrm{~nm}-\mathrm{rad})$ & Vertical emittance & 0.0079 \\
$\sigma_{z}(\mathrm{~mm})$ & Bunch length & 5 \\
$I(\mathrm{~A})$ & Total current & 1.5 \\
$n_{b}$ & Number of bunches & 3200 \\
$T_{l}$ (minute) & Beam lifetime & 19.4 \\
\hline \hline
\end{tabular}

TABLE X: PEP-X main parameters. Note that the emittances include the contribution from the intra-beam scattering assuming $5.5 \%$ of coupling.

Many results of the study should be considered preliminary given the uncertainties of inputs that went into the calculation. For example, the broad-band wakefield, based on the LER of PEP-II, serves only as a rough approximation of the impedance model for the new machine. Clearly, many more investigations are necessary to firm up those calculations and to optimize the design.

The study also provides us with many suggestions of how to improve the design: 
- Introduce third-harmonics cavities to lengthen the bunch and therefore to further reduce the effect of intra-beam scattering and to mitigate the microwave instability

- A faster feedback system in the transverse planes may be necessary to control the fast-ion stability and multi-bunch instability due to the resistive-wall impedance

- Increase the momentum compaction factor to mitigate the microwave instability

\section{Acknowledgments}

We would like to thank other members in the PEP-X study group for many helpful discussions. This work was supported by the Department of Energy under Contract Number: DE-AC02-76SF00515.

[1] "PEP-II: An Asymmetric B Factory", Conceptual Design Report, SLAC-418 (1993).

[2] L.C. Teng, Fermilab Report No. TM-1269 (1984).

[3] M. Sommer, LAL/RT/83-15 (1983).

[4] Helmut Wiedemann, Particle Accelerator Physics, Vol. I, (Springer-Verlag, Berlin Heidelberg, 1993).

[5] H. Grote and F.C. Iselin, CERN Report No. CERN/SL/90-12 (AP) (1994).

[6] Z. Huang et. al, SLAC-PUB-12858, to be published in Nucl. Instrum. Method A (2008).

[7] Y. Cai, M. Donald, I. Irwin, Y. Yan, SLAC-PUB-7642 (1997).

[8] K. Bane, in Proceedings of 8th European Particle Accelerator Conference, Paris, (2002).

[9] J.D. Bjorken and S.K. Mtingwa, Particle Accelerators 13, 115 (1983). 
[10] T. Raubenheimer, Particle Accelerators 45, 111 (1994).

[11] K. Kubo and K. Oide, Phys. Rev. ST-AB 4, 124401 (2001).

[12] H. Brück, Accelérateurs Circulaires de Particules, PUF (1966).

[13] A. Piwinski, in Handbook of Accelerator Physics, 3rd Printing, (World Scientific, New Jersey, 2006) p. 142.

[14] S. Heifets, A. Chao, E. Daly, K. Ko, N. Kurita, X. Lin, C. Ng, M. Nordby, C. Perkins, J. Seeman, et al., SLAC-AP-99 (1995).

[15] S. Heifets, C. Ng, A. Novokhatski, and S. Weathersby, SLAC-PUB-10837 (2004).

[16] G. Stupakov (2006), presented at ILC Damping Rings R\&D Workshop, September 26-28, 2006 at Cornell University.

[17] G. Stupakov and S. Heifets, Phys. Rev. ST Accel. Beams 5, 054402 (2002).

[18] R. L. Warnock, SLAC-PUB-5375 (1990).

[19] A. W. Chao, Physics of Collective Beam Instabilities in High Energy Accelerators (Wiley, New York, 1993).

[20] T. Raubenheimer and F. Zimmermann, Phys. Rev. E52, No. 5, 5487 (1995).

[21] G. Stupakov, Proc. Int. Workshop on Collective Effects and Impedance for B-Factories KEK Proc. 96-6, p243 (1996).

[22] L. Wang, Y. Cai and T. Raubenheimer, in Proceedings of Particle Accelerator Conference, Albuquerque, New Mexico, (2007). 